\title{
Intranuclear cascade model for a comprehensive description of spallation reaction data
}

\author{
A. Boudard, ${ }^{1}$ J. Cugnon, ${ }^{2}$ S. Leray, ${ }^{1}$ and C. Volant ${ }^{1}$ \\ ${ }^{1}$ DAPNIA/SPhN, CEA/Saclay, F-91191 Gif-sur-Yvette Cedex, France \\ ${ }^{2}$ Université de Liège, Institut de Physique B5, Sart Tilman, B-4000 LIEGE 1, Belgium \\ (Received 12 December 2001; published 29 October 2002)
}

\begin{abstract}
A new version of the Liège intranuclear cascade (INC) model is proposed for the description of spallation reactions. Compared to the previous version, it incorporates new features: (i) it can accommodate a diffuse nuclear surface, (ii) the treatment of the Pauli blocking effect is improved, removing unphysical features linked with the use of statistical blocking factors, (iii) collisions between moving spectator nucleons are explicitly suppressed, (iv) pion dynamics is improved, especially concerning the delta lifetime, (v) it can accommodate light ions as incoming projecticles, (vi) the remnant angular momentum is included in the output of the model. Another important feature is the self-consistent determination of the stopping time, i.e., the time at which the INC calculation is terminated and coupled to evaporation. The predictions of the model, used with the Schmidt evaporation code, are tested against a large body of experimental data, in the $200-\mathrm{MeV}-2-\mathrm{GeV}$ range for incident energy per nucleon, including total reaction cross sections, neutron, proton, pion, and composite double differential cross sections, particle multiplicities, residue mass and charge distributions, and residue recoil velocity distributions. Good agreement is generally obtained without additional varying parameters. It is shown that the introduction of a diffuse surface considerably improves the description of the total reaction cross sections, of the intensity of the quasielastic peak in proton and neutron double differential cross sections and of the residue production yield for isotopes close to the target. High energy neutron spectra are found to be sensitive to details of the deuteron structure in deuteron-induced reactions. The shape of the fragmentation peaks in residue mass spectra is shown to be closely related to the shape of the distribution of the excitation energy left after the cascade stage. The longitudinal residue recoil velocity and its fluctuations display typical random-walk characterics, which are interpreted as a direct consequence of the independence of successive binary collisions occurring during the cascade process and therefore provide a strong support of the basic hypotheses of the INC model. Small but systematic discrepancies between model predictions and experiment are identified and possible further improvements to reduce them are discussed. The influence of the evaporation model is investigated. A comparison with similar approaches is presented.
\end{abstract}

DOI: 10.1103/PhysRevC.66.044615 PACS number(s): 25.40.Sc, 25.45.-z, 25.85.Ge, 28.20.-v

\section{INTRODUCTION}

There is a strongly renewed interest in the study of spallation reactions, largely triggered by the advent of the concept of accelerator-driven systems (ADS's). Such devices, consisting of a subcritical assembly coupled to an accelerator of particles, may play an important role in the future management of nuclear waste $[1,2]$. Many projects of ADS have appeared around the world. The interest in spallation reactions has been renewed also, and perhaps more importantly, by the current projects of intense, pulsed, spallation neutron sources (see, e.g., Ref. [3]). One has also to notice the growing development of radioactive beams: many radioactive nuclei can be produced by bombarding a thick spallation target with high energy particles [4]. To be complete, let us also mention the relevance of spallation reactions in astrophysics [5], cosmic ray physics [6], and planetary science [7].

The technological applications mentioned above require an optimization of the so-called spallation targets or spallation sources. Due to the large number of parameters characterizing these sources, this optimization cannot be done by an empirical trial and error method. One has to rely on simulations. Powerful tools are thus needed to describe the series of processes that occur when high energy particles hit and propagate inside a macroscopic piece of matter. In turn, this description requires an accurate simulation of what happens at the microscopic level during a particle-nucleus reaction. For incident particle energies not exceeding $\sim 200 \mathrm{MeV}$, one may have recourse to cross-section libraries, which are currently generated [8]. Above this energy, the number of open channels become prohibitive and direct use of reliable models is necessary, the most appropriate one being the intranuclear cascade (INC) plus evaporation model. Furthermore, it is generally considered that the lower limit of validity of the INC model corresponds more or less to the energy mentioned above (see, however, Refs. [9,10]). There is thus a need to assess the accuracy of the INC and evaporation models, in the $200-\mathrm{MeV}-2-\mathrm{GeV}$ energy domain, and to determine whether these models can be improved. Such an effort concerning the Liège INC model is reported on in this paper. This model was first developed for heavy-ion collisions and was quite instrumental to pin down the physical aspects of these collisions in the $\mathrm{GeV}$ range [11]. Later, this model was used for antiproton-nucleus interactions [12]. Finally, a version of the model was constructed for the nucleon-nucleus case [13]. Although it helped to clarify many aspects of the reaction mechanism (in particular, the relevance of nuclear matter stopping power was pointed out and its evaluation was done for the first time), this version was rather crude. An improved version of the model was proposed a few years ago [14], which basically introduced a better parametrization of baryon-baryon collisions and a self-consistent determination 
of the stopping time, i.e., the time at which the cascade model should be stopped to give way to the evaporation step (see below). It has been shown in Ref. [14] that this version of the Liège INC model is able to reproduce with a reasonable accuracy the neutron double differential cross sections and the scarce and imprecise (at that time) residue cross sections. However, systematic discrepancies were observed, which were attributed to the presence of a sharp nuclear surface. It is the purpose of this paper to present a new version of the Liège INC model which removes this deficiency. We also introduce several other improvements of the physical content of the model. They are described below, the most important one consisting in a better treatment of the Pauli principle.

Our strategy is to improve the quasiclassical treatment of the physics embodied by the model without relying on too many free parameters. Most of the parameters describing this physics are either taken from phenomenology (like nuclear radii) or have been determined by previous studies (like the parameters of the Pauli blocking). We accept only two free parameters and we will show that, using their most likely value, we can describe a large set of experimental data with, frequently, high accuracy.

As will be explained below, the present approach offers several advantages in comparison with other currently used INC models, as the Bertini [16] and the ISABEL [17,18] models: (i) it includes a realistic target density distribution, (ii) the fate of all particles is followed as time evolves, (iii) it incorporates a sort of self-consistent determination of the stopping time, (iv) Pauli blocking is implemented consistently with the progressive depletion of the Fermi sphere, (v) it is able to successfully describe at the same time a large set of various observables. Furthermore, it is almost parameter free, in the sense outlined above.

The paper is divided as follows. In Sec. II, we describe the Liège INC model with emphasis on the new features. We also comment on the various conservation laws of the model and on the free parameters. Section III is devoted to an extensive comparison with a representative panel of experimental data: total reaction cross section, neutron, proton and pion double differential cross sections, residue production cross sections, residue recoil velocity distributions, etc., mainly for proton-induced, but also for deuteron-induced, reactions. In Sec. IV, we critically examine the effects of changing the value of the stopping time. We also pay some attention to the influence on the observables of the implementation of the Pauli blocking and of the evaporation model. Section V is devoted to a discussion of the physical aspects of the model, to an identification of the remaining deficiencies, and to the possible ways of removing them. In Sec. VI, we present a short comparison with similar works. Finally, Sec. VII contains our conclusion.

\section{LIÈGE INC MODEL: PRESENT VERSION}

\section{A. Short account of the standard Liège INC model}

The standard Liège INC model is described in detail in Refs. $[14,15]$ and in references cited therein. It is sufficient here to recall that the collision mechanism is assumed to proceed from a succession of binary collisions (and decays) well separated in space and time. The fate of all particles is followed as time evolves. The particles travel along straightline trajectories until two of them reach their minimum distance of approach, in which case they can be scattered provided the value of this distance is small enough, or until they hit the border of the potential well, supposed to describe the nuclear target mean field. Additional features are (i) initial positions of target nucleons are taken at random in the spherical nuclear target volume with a sharp surface; (ii) initial momenta are generated stochastically in a Fermi sphere; (iii) relativistic kinematics is used; (iv) inelastic collisions, pion production, and absorption are supposed to proceed from the following reactions:

$$
N N \rightleftharpoons N \Delta, \quad \Delta \rightleftharpoons \pi N
$$

(v) isospin degrees of freedom are introduced for all types of particles and isospin symmetry is respected; (vi) the Pauli principle is enforced by means of statistical blocking factors.

One of the advantages of this model comes from straightline trajectories. At any time, any pair of particles can be checked and the time span necessary to reach their minimum relative distance of approach can be evaluated. As a consequence the next collision can be foreseen and particles can be propagated at once untill the time of this encounter.

The stochastic realization of the model brings disturbing features. The random generation of the initial state (in spheres in $\vec{r}$ and $\vec{p}$ spaces) gives rise to fluctuations around an average uniform phase-space population. As a result, the phase-space occupation probability, when tested by summing particles in a small "measuring" volume, may take values smaller (or larger) than unity. This spurious depletion of the Fermi sea and the stochastic treatment of the Fermi blocking may generate unphysical effects. Let us suppose there is a spurious vacancy around some momentum $p_{0}$. As the Pauli blocking is applied stochastically, the incoming particle colliding with a target nucleon of momentum larger than $p_{0}$ may leave this nucleon in the vacancy: the target has so lost energy, i.e., has acquired a negative excitation energy! Although on the average the treatment of the Pauli blocking is presumably correct and although the percentage of events with final negative excitation energy is rather small, this weakness needs to be cured. In Refs. [14,19-21], events with a final negative excitation energy were simply considered as zero excitation energy events, whereas in subsequent uses of the Liège standard model, the cascade events were terminated just before the first collision which could lead to a negative excitation energy [22-24]. These two choices are referred to the INCL2 and INCL3 versions of the Liège INC model, respectively. A more satisfactory treatment is needed and is presented below.

\section{B. Description of the new ingredients}

\section{Nuclear surface}

The most important of these new ingredients is the introduction of a diffuse nuclear surface, corresponding to a Saxon-Woods density distribution, up to maximum distance $R_{\text {max }}$, fixed to $R_{0}+8 a$ : 


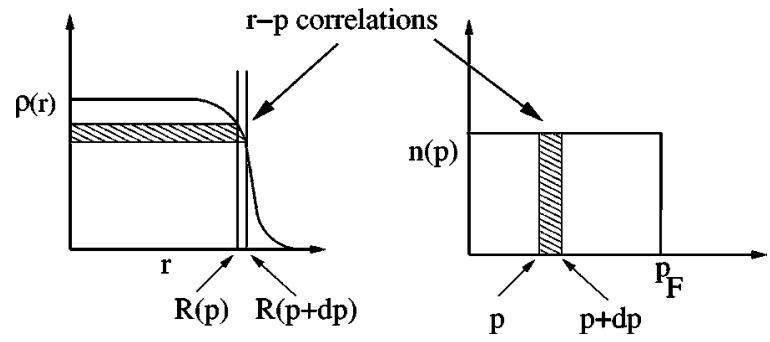

FIG. 1. Illustration of the correlation between the spatial and momentum distributions implied by the phase space distribution [Eq. (2)]. Particles with momentum between $p$ and $p+d p$ are those that can move up to a maximum radial distance between $R(p)$ and $R(p+d p)$. Consequently, the numbers of nucleons corresponding to the shaded areas should be the same. This defines the function $R(p)$. See text and Appendix for details.

$$
\rho(r)= \begin{cases}\frac{\rho_{0}}{1+\exp \left(\frac{r-R_{0}}{a}\right)} & \text { for } r<R_{\max } \\ 0 & \text { for } r>R_{\max } .\end{cases}
$$

The values of $R_{0}$ and $a$ are taken from electron scattering measurements and parametrized, for convenience, from $A l$ to $U, \quad$ as $\quad R_{0}=\left(2.745 \times 10^{-4} A_{T}+1.063\right) A_{T}^{1 / 3} \mathrm{fm}, \quad a=0.510$ $+1.63 \times 10^{-4} A_{T}$ fm [in the numerical code, other values, as well as another shape for $\rho(r)$, can optionally be introduced]. The quantity $\rho_{0}$ is such that the distribution is normalized to $A_{T}$, the target mass number. The momentum distribution is kept as a uniform Fermi sphere with Fermi momentum $p_{F}$.

Nucleons with a large momentum (in the central part of the nucleus) are expected to travel farther out than those with a small momentum. These $r-p$ correlations make it impossible to generate the $r$ and $p$ distributions independently. Furthermore, these distributions should remain constant when the target is left alone, supposing nucleons evolve freely in an average nuclear potential. These two requirements can be fulfilled by placing nucleons of momentum $p$ in a square well of depth $V_{0}$ with a momentum dependent well radius $R(p)$ suitably chosen, as shown in Fig. 1: nucleons with momentum between $p$ and $p+d p$ will be characterized by a constant uniform probability within a sphere of radius $R(p)$, once they are initially generated with this property. They contribute to the density profile by the layer shown by the shaded area on the left-hand side. This implies that the number of nucleons with momentum between $p$ and $p+d p$ is the same as the number of nucleons populating this layer, which yields

$$
\begin{aligned}
A_{T} \frac{4 \pi p^{2} d p}{\frac{4 \pi}{3} p_{F}^{3}} & =\frac{4 \pi}{3} R^{3}(p) d \rho \\
& =-\left.\frac{4 \pi}{3} R^{3}(p) \frac{d \rho(r)}{d r}\right|_{r=R(p)} R^{\prime}(p) d p
\end{aligned}
$$

where $d \rho$ is the thickness of the density layer. With $R(0)$ $=0$, this relation is equivalent to

$$
\left(\frac{p}{p_{F}}\right)^{3}=-\frac{4 \pi}{3 A_{T}} \int_{0}^{R(p)} \frac{d \rho(r)}{d r} r^{3} d r .
$$

The initial position and momentum of any target nucleon are generated as follows: $\vec{p}$ is taken at random in a sphere of radius $p_{F}, R(p)$ is calculated by relation (4), and $\vec{r}$ is chosen at random in a sphere of radius $R(p)$. This is equivalent to taking $\vec{r}, \vec{p}$ at random according to the joint probability distribution

$$
\frac{d n}{d^{3} \vec{r} d^{3} \vec{p}}=f(\vec{r}, \vec{p})=A_{T} \frac{\theta(R(p)-r) \theta\left(p_{F}-p\right)}{\left(\frac{4 \pi}{3}\right)^{2} R^{3}(p) p_{F}^{3}}
$$

where $\theta(x)$ is the Heaviside function. It is explicitly shown in the Appendix that $R(p)$ is an increasing function of $p$, with $R(0)=0$ and $R\left(p_{F}\right)=R_{\text {max }}$, and that, after integration on the relevant variables, the joint distribution in Eq. (5) corresponds to the spatial density $\rho(\vec{r})$ and to the sharp Fermi sphere momentum distribution:

$$
\begin{gathered}
\int f(\vec{r}, \vec{p}) d^{3} p=\rho(\vec{r}), \\
\int f(\vec{r}, \vec{p}) d^{3} r=A_{T} \frac{\theta\left(p_{F}-p\right)}{\frac{4 \pi}{3} p_{F}^{3}} .
\end{gathered}
$$

The procedure outlined above is at variance with the one used in many transport models [25], where nucleons are placed in a potential with a Saxon-Woods or similar shape. We discarded this choice because it removes the possibility of propagating particles in a single step between collisions (or reflections), a very appealing feature of Liège model, since their momentum is going to change in between. Note, however, that, within our approach, only fast particles in the full strength potential can be found in the fringes of the target nucleus, which is unphysical or at least in contradistinction with semiclassical models of the nuclear phase-space density. We recall, however, that the validity of these models just breaks down in the classically forbidden regions, i.e., basically in outer surface [26].

\section{Consistent dynamical Pauli blocking}

The Pauli blocking is often enforced (see, e.g., Ref. [16]) by allowing only collisions leading to final states with nucleon momenta above the Fermi momentum. This neglects the depletion of the Fermi sphere. Furthermore, the collision process also induces a temporary depletion of the spatial density. This possibility is accounted for in the INC models of Refs. $[17,18]$ by punching holes in the continuous density profile. However, in principle, the Pauli blocking should operate in phase space. This is realized in the standard Liège model (as in many heavy-ion collision models) as follows: if two nucleons $i$ and $j$ are going to suffer a collision at posi- 
tions $\vec{r}_{i(j)}$ leading to a final state with momenta $\vec{p}_{i(j)}$, the phase-space occupation probabilities $f_{i}$ are evaluated by counting nearby nucleons in a small phase-space volume,

$$
\begin{aligned}
f_{i}= & \frac{1}{2} \frac{(2 \pi \hbar)^{3}}{\frac{4 \pi}{3} r_{P B}^{3} \frac{4 \pi}{3} p_{P B}^{3}} \sum_{k \neq i} \theta\left(r_{P B}-\left|\vec{r}_{k}-\vec{r}_{i}\right|\right) \\
& \times \theta\left(p_{P B}-\left|\vec{p}_{k}-\vec{p}_{i}\right|\right)
\end{aligned}
$$

where the sum is limited to particles $k$ with the same isospin component as particle $i$ (or $j$ ). The factor $1 / 2$ is introduced because spin components are ignored. The collision between $i$ and $j$ is allowed or forbidden following the comparison of a random number with the product $\left(1-f_{i}\right)\left(1-f_{j}\right)$. Pauli blocking is not applied to $\Delta$ particles because their density is always very small. On the other hand, it is enforced for nucleons resulting from $\Delta$ decays. The parameters $r_{P B}$ and $p_{P B}$ should not be taken too small, otherwise $f_{i}$ is going to be always vanishingly small, nor too large, otherwise the details of the phase-space occupation can be missed. There is no a priori criterion for the appropriate choice of these parameters. In practice, $r_{P B}$ and $p_{P B}$ are taken just large enough for results to be roughly insensitive to moderate modifications of their values (see Ref. [14]). In this work, $r_{P B}=3.18 \mathrm{fm}$ and $p_{P B}=200 \mathrm{MeV} / c$, which corresponds to a measuring volume of $\sim 2.3$ natural units of phase space.

The interplay between this stochastic implementation of the Pauli blocking and the fluctuations of the phase-space occupation in the initial state, inherent in the stochastic generation of the latter, may introduce unphysical results unless sufficient care is exercised, as we have already noticed. To remedy this situation, while still taking account of the depletion of the Fermi sphere, we add here the following procedure: we evaluate the energy contained in the Fermi sphere, i.e., the kinetic energy of particles with $p<p_{F}$, and we do not allow a collision if this energy is going to be smaller than the actual (instantaneous) Fermi-gas minimum energy. A mathematical formulation is given below in expression (22).

\section{Division into participants and spectators}

Participants are defined as particles having collided with at least one other participant, the incident particles being the only participants at the beginning. Spectators are the other particles. All particles are moving but, contrary to previous versions of the model, collisions are forbidden between spectators. Propagation of spectators provides a natural evolution of the early perturbations of the spatial density. This procedure is more satisfactory than the corresponding one in INC models with a continuous density distribution, which implies some ad hoc filling of the holes punched by the participants [17]. In addition, forbidding collisions between spectators eliminates the "spontaneous boiling" of the Fermi sea: in absence of this prescription, nucleons close to the Fermi surface can, again because of the same reasons as those discussed in the previous paragraph, gain energy through collisions between themselves at the expense of others, and escape from the target even if the latter is left alone. In previous versions of the Liège model, this spurious evaporation could lead to an increase of a few percent of the neutron emission in the cascade [27]. Of course, it had no practical influence on the neutron energy outflow.

\section{Improvement of pion dynamics}

Pions and deltas are supposed to appear and disappear through the $N N \rightleftharpoons N \Delta$ and $\Delta \rightleftharpoons \pi N$ reactions. We recall that for $\pi N$ interaction, we use experimental cross sections, including nonresonant scattering, but we treat the latter as proceeding through the formation of a $\Delta$ with a very short lifetime; inelastic $\pi N$ scattering is neglected for convenience [14].

In the $N N \rightarrow N \Delta$ process, the " $\Delta$ particle" is given a mass $m_{\Delta}$, taken at random in the distribution

$$
f\left(m_{\Delta}\right)=F_{N} \frac{q^{3}}{q^{3}+q_{0}^{3}} \frac{1}{1+4\left(\frac{m_{\Delta}-m_{\Delta}^{0}}{\Gamma_{0}}\right)^{2}},
$$

with

$$
q^{2}=\frac{\left[m_{\Delta}^{2}-\left(m_{N}-m_{\pi}\right)^{2}\right]\left[m_{\Delta}^{2}-\left(m_{N}+m_{\pi}\right)^{2}\right]}{4 m_{\Delta}^{2}}
$$

subject to kinematical constraints, i.e., with $m_{\Delta}$ lying in the interval $\left[m_{N}+m_{\pi}, \sqrt{s}-m_{N}\right], \sqrt{s}$ being the c.m. energy of the collision, and consistent with energy-momentum conservation. The quantity $F_{N}$ in Eq. (9) is the normalization constant. The parameters are $q_{0}=0.18 \mathrm{GeV}, m_{\Delta}^{0}=1.215 \mathrm{GeV}$, and $\Gamma_{0}=0.13 \mathrm{GeV}$. The introduction of the $q$-dependent factor is required by the fit of $N N \rightarrow N \Delta$ data [28] and can be justified as follows: a $\Delta$ resonance is a correlated pionnucleon system and the phase space of the latter system is considerably reduced when its c.m. energy is low.

In previous versions, the $\Delta$ particles were given an average intrinsic lifetime $\tau_{\Delta}=\tau_{0}=\hbar / \Gamma_{0}$ (in proper time and disregarding Pauli blocking). We here adopt

$$
\frac{1}{\tau_{\Delta}}=\frac{q^{3}}{q^{3}+q_{0}^{3}} \frac{1}{\tau_{0}}
$$

This is justified as, if the $\Delta$ resonance is going to decay in a $\pi N$ pair with low energy (which is the case for small $m_{\Delta}$ in our classical picture), the decay width is considerably diminished due the reduction of phase space. This modification was already advocated in Ref. [18].

The $N \Delta \rightarrow N N$ cross section is usually taken from detailed balance in the Liège INC model. However, detailed balance strictly holds for stable particles only and should be corrected for unstable ones. Let us here just make a few qualitative considerations on how this correction can be established. The $N N \rightarrow N \Delta$ cross section that is to be introduced in a simulation like ours has to describe the real probability to produce a $\Delta$ at the end of the collision. It is not the cross section entering the detailed balance relation, as a loss of flux occurs even during the collision, because of the coupling to 
TABLE I. Parameters of the Gaussian forms used to describe radial distance and momentum distributions in light ions.

\begin{tabular}{lcc}
\hline \hline Particle & $\sqrt{\left\langle r^{2}\right\rangle}(\mathrm{fm})$ & $\sqrt{\left\langle p^{2}\right\rangle}(\mathrm{MeV} / c)$ \\
\hline$d$ & 1.91 & 77 \\
$t$ & 1.8 & 110 \\
${ }^{3} \mathrm{He}$ & 1.8 & 110 \\
${ }^{4} \mathrm{He}$ & 1.63 & 153 \\
\hline \hline
\end{tabular}

the pion-nucleon channel. In fact the "theoretical" fluxconserving cross section (i.e., the cross section that could be defined by artificially cutting down the coupling to the $\pi N$ channel) is smaller. The converse is true for the $N \Delta \rightarrow N N$ cross section. Flux-conserving cross sections are related by detailed balance. A modified relation applies to effective cross sections. The relevant parameter here is the ratio between the (average) collision time $t_{\text {coll }}$ and the $\Delta$ lifetime $\tau_{\Delta}$. If this ratio is very small, one is close to the case of stable particles and the correction should vanish. On the contrary, if $t_{\text {coll }} / \tau_{\Delta}$ is very large, the correction should be very large. Therefore it is reasonable to adopt the following modification:

$$
\sigma_{N \Delta \rightarrow N N}=g \sigma_{N N \rightarrow N \Delta} e^{t_{c o l l} / \tau_{\Delta}}
$$

where $g$ is the usual ratio of phase-space factors and degeneracies. The collision time is not a well-known quantity, but one may consider that it is of the order of $1-2 \mathrm{fm} / \mathrm{c}$. We adopt a factor 3 for the correction term, i.e., for the exponential in the last equation. Such a correction factor was already proposed in Ref. [29] on a phenomelogical basis as a way to correctly describe pion absorption on nuclei.

\section{Extension to incident light clusters}

Extension to incident clusters of nucleons (we limit ourselves to deuterons, tritons, ${ }^{3} \mathrm{He}$ and ${ }^{4} \mathrm{He}$ ions) is rather natural in our model, as the fate of all particles is followed at the same time. It is therefore sufficient to generate the initial distribution of nucleons inside the incoming cluster. Due to the small size of these clusters, it is not appropriate to use a distribution of the form [Eq. (2)] for their initial state. We use in fact a Gaussian shape for the spatial distribution with a width which is determined by the charge r.m.s. radius. We also do the same for the momentum distribution, with widths taken from literature [30-33] (see Table I), except for the deuteron. This loosely bound system requires a more careful microscopic treatment. We used the modulus squared of the wave function in momentum space, as calculated with the Paris potential [34].

We do not introduce a nuclear mean field inside the incoming ion. This approximation is reasonable in view of their weak binding (except for ${ }^{4} \mathrm{He}$ ). To correct for the latter in the energy balance, we decrease the incident kinetic energy by an amount equal to the binding energy in order to have the correct total incident energy.

\section{Conservation laws}

It is instructive to analyze how our INC model handles conservation laws. In the energy range under consideration, the most important conservation laws can be formulated as follows (in the lab system):

$$
\begin{gathered}
A_{P}+A_{T}=A_{e j}+A_{r e m}, \\
Z_{P}+Z_{T}=Z_{e j}+Z_{\pi}+Z_{r e m}, \\
T_{l a b}=K_{e j}+W_{\pi}+E_{r e c}+E^{*}+S, \\
\vec{p}_{l a b}=\vec{p}_{e j}+\vec{p}_{\pi}+\vec{p}_{r e m}, \\
\vec{\ell}=\vec{\ell}_{e j}+\vec{\ell}_{\pi}+\vec{\ell}_{r e m}+\vec{\ell}^{*}
\end{gathered}
$$

for baryon number, charge, energy, momentum, and angular momentum, respectively. We consider a projectile $P$ colliding with a target $T$ and generating (baryonic) ejectiles, pions, and a remnant (in this paper we distinguish between the remnant, which is the remaining part of the target up to the end of the cascade and the residue, which refers to the end of the evaporation stage). In Eq. (15), $K_{e j}$ is the kinetic energy of the ejectiles, $W_{\pi}$ is the total energy of the pions, $E_{r e c}$ is the recoil energy of the remnant, $E^{*}$ is the remnant excitation energy, and $S$ is the separation energy, i.e., the minimum energy needed to remove all ejectiles and pions from the target nucleus ground state. In the other equations, the indices have similar meaning. In Eq. (17), $\vec{\ell}$ is the angular momentum of the incident particle, $\vec{\ell}_{\text {rem }}$ is the angular momentum corresponding to the c.m. motion of the remnant, and $\vec{\ell} *$ is the intrinsic angular momentum of the remnant. Other notations in Eqs. (13)-(17) are self-explanatory.

In our INC model, ejectiles correspond to baryons outside the potential well and there is no interaction between particles outside the potential well and no interaction between them and the remnant. We can thus be more specific about the formulation of conservation laws (13)-(17). First, they hold at any time and not only asymptotically. Second, the energy conservation law can be given another expression in term of kinetic energies. Indeed, one can write, for any moment of the collision process,

$$
T_{l a b}+\sum_{i \in A_{T}}\left(T_{i}^{0}-V_{0}\right)=\sum_{j=1}^{A_{e j}} \bar{T}_{j}+W_{\pi}+\sum_{i \in A_{r e m}}\left(\bar{T}_{i}-V_{0}\right),
$$

where the three summations run over the target nucleons in the initial state, the ejectiles, and the baryons remaining in the potential well (the remnant), respectively. The quantity $W_{\pi}$ denotes the total energy of the pions, irrespective of their location inside or outside the target nucleus. The bar in Eq. (18) means that the $\Delta$-nucleon mass difference is added to the kinetic energy of the $\Delta$ 's (which ultimately decay, but can be present at any finite time). In our approach, the remnant is "attached" to the fixed potential well, and the recoil of the remnant is not explicitly taken into account. On the other hand, one can rewrite Eq. (18) as 


$$
\begin{aligned}
T_{l a b}= & \sum_{j=1}^{A_{e j}} \bar{T}_{j}+W_{\pi}+\left\{\sum_{i \in A_{\text {rem }}} \bar{T}_{i}-\left[\sum_{i \in A_{T}} T_{i}^{0}-\left(A_{T}\right.\right.\right. \\
& \left.\left.\left.-A_{\text {rem }}\right) T_{F}\right]\right\}+\left(A_{T}-A_{r e m}\right)\left(V_{0}-T_{F}\right),
\end{aligned}
$$

where $T_{F}$ is the Fermi kinetic energy. The quantity

$$
S=\left(A_{T}-A_{\text {rem }}\right)\left(V_{0}-T_{F}\right)
$$

represents the minimum energy required to remove $A_{T}$ $-A_{\text {rem }}$ nucleons from the target in our model and can thus be considered as the (total) separation energy. Therefore the quantity

$$
E^{*}=\sum_{i \in A_{\text {rem }}} \bar{T}_{i}-\left[\sum_{i \in A_{T}} T_{i}^{0}-\left(A_{T}-A_{\text {rem }}\right) T_{F}\right]
$$

is to be interpreted as the excitation energy, since the expression in the squared bracket represents the minimum energy of the actual remnant (in our model). One could refine the model by taking $S$ from binding-energy tables, but we prefer to keep it as part of our model, allowing some possible tuning by an appropriate choice of $V_{0}$. We can also split $E^{*}$ into two pieces:

$$
\begin{aligned}
E^{*}= & \sum_{\substack{i \in A_{\text {rem }} \\
p_{i}>p_{F}}}\left(\bar{T}_{i}-T_{F}\right)+\left\{\sum_{\substack{i \in A_{\text {rem }} \\
p_{i} \leqslant p_{F}}} \bar{T}_{i}-\left[\sum_{i \in A_{T}} T_{i}^{0}-\left(A_{T}\right.\right.\right. \\
& \left.\left.\left.-A_{\text {rem }}^{F}\right) T_{F}\right]\right\},
\end{aligned}
$$

where $A_{r e m}^{F}$ is the number of nucleons in the remnant with momentum less than $p_{F}$. The quantity in the squared bracket is the ground state of the actual Fermi sea. It differs from the ground state of the actual remnant as the latter can contain more nucleons $\left(A_{\text {rem }} \geqslant A_{r e m}^{F}\right)$. The quantity inside the large parentheses represents the excitation energy due to the rearrangement of the level occupations inside the original Fermi sphere, made possible by the depletion of the latter. As explained above, this quantity is checked at any possible baryon-baryon collision and is not allowed to become negative.

In our model, conservation laws (13) and (14) are, of course, exactly fulfilled and energy is conserved: relations (18) and (15), with $E_{r e c}=0$ and $S$ and $E^{*}$ defined by Eqs. (20) and (21), respectively, are fulfilled with a numerical accuracy always better than a few $\mathrm{keV}$, of the order of $1 \mathrm{keV}$ on the average. On the other hand, momentum and angular momentum are not conserved, when in Eqs. (16) and (17) the quantities $\vec{p}_{r e m}, \vec{\ell}_{r e m}$, and $\vec{\ell}^{*}$ are evaluated by summing momenta or angular momenta of particles inside the remnant. Momentum is conserved in baryon-baryon collisions, but not in reflections from and transmissions through the potential border. If it were, for instance by making the remnant recoil as a whole, the latter would do it with a velocity which is roughly the velocity of the ejected particle divided by the remnant mass number. Therefore neglecting this recoil generates a relative error in the evaluation of $\vec{p}_{e j}$ of the order of $1 / A_{\text {rem }}$. It is thus legitimate to consider that our model calculates $\vec{p}_{e j}$ and $\vec{p}_{\pi}$ with little error and to evaluate the remnant momentum by difference,

$$
\overrightarrow{\tilde{p}}_{r e m}=\vec{p}_{l a b}-\vec{p}_{e j}-\vec{p}_{\pi},
$$

with the right-hand side (r.h.s.) quantities evaluated in the model. It should be stressed that, even if this quantity can be a large fraction of $p_{l a b}$, the corresponding recoil energy (here given in its nonrelativistic form),

$$
\widetilde{E}_{r e c}=\frac{\tilde{p}_{r e m}^{2}}{2 m_{N} A_{r e m}}
$$

is always very small compared to the other terms of Eq. (15) (see below). Therefore we can slightly correct the final calculated value of the ejectile momenta (including pions), by means of a multiplicative factor $f_{c}$, in order to accommodate the recoil energy, while still preserving the energy balance and the consistency of Eqs. (23) and (24). This can be done iteratively, by first multiplying the ejectile momenta by $f_{c}^{0}$, in order to insert the recoil energy in the r.h.s. of Eq. (15), still preserving the equality. The quantities $\tilde{p}_{r e m}$ and $\widetilde{E}_{r e c}$ are reevaluated with the corrected momenta. The latter are multiplied by $f_{c}^{1}$ in order to insert the newly evaluated recoil energy in the r.h.s. of Eq. (15), and so on. This procedure is stopped at the first two indicated steps, since the factor $f_{c}^{0}$ turns out to be very close to unity, in all cases.

Angular momentum is not conserved in our INC model because of transmission through the surface (nucleon momentum is changed) and also because the scattering plane is not preserved in binary collisions. This matter is discussed in detail in Refs. [14,27], where an elaborate prescription is proposed to conserve angular momentum in binary collisions. It is also shown in these references that the calculated angular momentum transfer is roughly the same with or without this prescription. The reason is that large angular momentum transfers come from intermediate impact parameter collisions, where fast ejectiles are emitted roughly (on the average at least) along the incident direction with less momentum than the incident particle: angular momentum transfer comes from momentum transfer to the ejected nucleons and not so much from the detail of the binary collision scenario. These considerations allow us to calculate the remnant internal angular momentum as the difference between initial and final angular momenta,

$$
\vec{\ell}^{*}=\vec{\ell}-\left(\vec{\ell}_{e j}+\vec{\ell}_{\pi}+\vec{\ell}_{r e m}\right)
$$

with a reasonable accuracy. The quantity $\vec{\ell}_{r e m}=\vec{R}_{r e m} \times \vec{p}_{r e m}$ (where $\vec{R}_{r e m}$ is the location of the barycenter of the remnant) is always a small fraction (about 10\%) of the average value of $\ell^{*}$. Keeping the latter as an output of the calculation will be of importance for the description of the fission yield and is better than ad hoc prescriptions. 

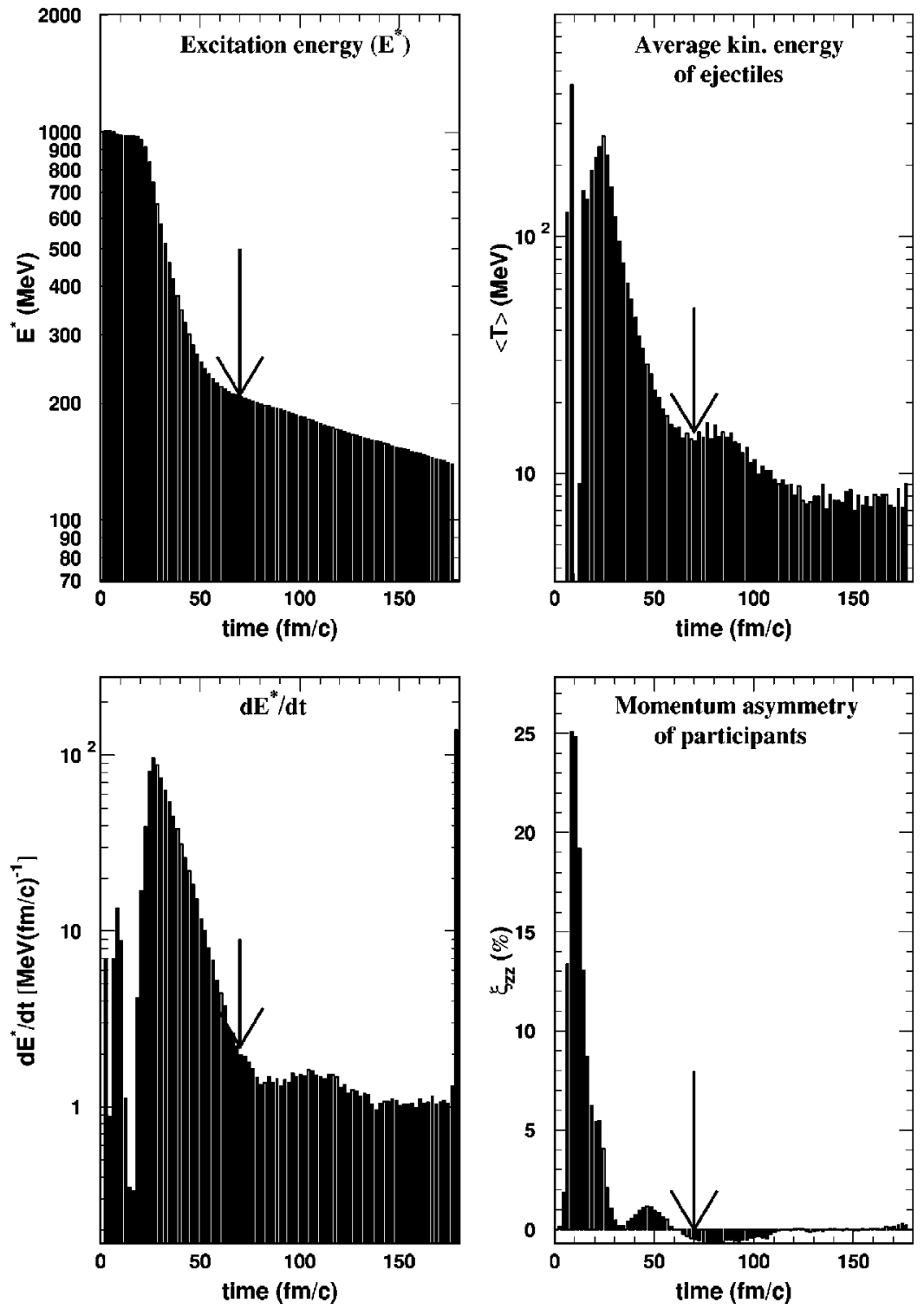

FIG. 2. Time variation of the average value of a few physical quantities, within our INC model. The panels refer, in a clockwise order, starting from the upper left, to the excitation energy, the average kinetic energy of the ejectiles, the asymmetry of the participant momentum distribution, and the time derivative of the excitation energy, respectively. The results correspond to collisions of $1-\mathrm{GeV}$ protons with $\mathrm{Pb}$ nuclei with an impact parameter of $4 \mathrm{fm}$. The arrows indicate the chosen stopping time.

\section{Stopping time}

As explained in Ref. [14], the stopping time $t_{\text {stop }}$, i.e., the time at which the cascade is stopped to give way to evaporation, is an important feature of our INC model. It turns out that the time evolution of the average (over runs) value of many physical quantities show, when the cascade is run for a long time, a phase of rapid variations, followed by a phase of much slower variations. In addition, the time of separation between the two phases is roughly the same for most of these physical quantities. These results enable us to define the stopping time more or less precisely as the common separation time of the phases of variation of the physical quantities. Figure 2 shows, for some typical case and a given impact parameter, the analysis of a few physical quantities: the excitation energy $E^{*}$, defined in Eq. (21), its time derivative, the mean kinetic energy of the ejected particles (i.e., the ratio $\left.T_{e j} / A_{e j}\right)$, and a quantity measuring the anisotropy of the momentum content of the participant baryons inside the target volume, namely,

$$
\xi_{z z}=\frac{\sigma_{z}^{2}-\frac{1}{2}\left(\sigma_{x}^{2}+\sigma_{y}^{2}\right)}{\sigma_{x}^{2}+\sigma_{y}^{2}+\sigma_{z}^{2}}
$$

where $z$ is the incident direction. The quantities $\sigma_{k}^{2}(k$ $=x, y, z)$ are defined by

$$
\begin{gathered}
\sigma_{k}^{2}=\sum_{i \in A_{r e m}}\left(p_{i k}-\left\langle p_{i k}\right\rangle\right)^{2}, \\
\left\langle p_{i k}\right\rangle=\frac{1}{A_{\text {rem }}} \sum_{i \in A_{r e m}} p_{i k} .
\end{gathered}
$$

In the early moments of the collision, $\xi_{z z}$ is different from zero because of the motion of the incident particle. It then decreases and tends to zero signalling that the system reaches a high degree of randomization. The quantity $E^{*}$ is large at the beginning of the collision, once the incident particle has penetrated the target. It then stays roughly constant for a while, then decreases with a high rate [35], corresponding 

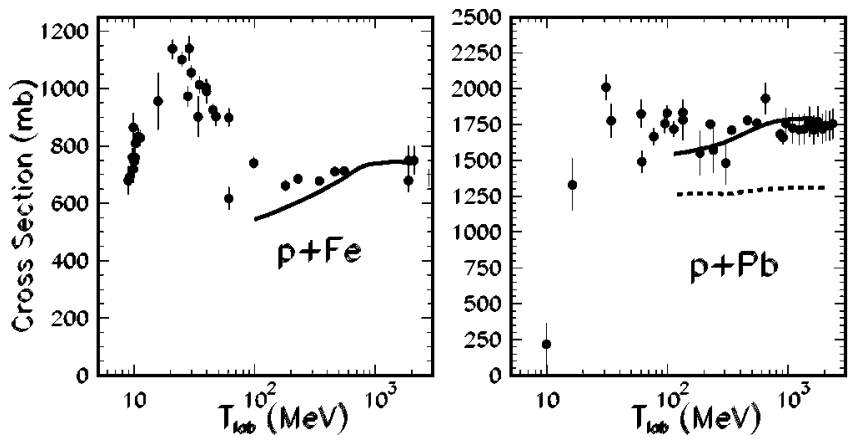

to the emission of fast particles, and undergoes a further decrease at a much lower pace, very much akin to an evaporation process. It is also manifest from Fig. 2 that the change of slope in the curve of $E^{*}$ is correlated with the change in the curve representing the average kinetic energy of the emitted particles: the latter quantity is rather large in the first phase, but reaches in the second phase a small and smoothly decreasing value, typical of the evaporative cooling of an equilibrated system. Although not shown in Fig. 2, change of rate of variation of other quantities like the number of participants and the total kinetic energy of the ejectiles also occurs at roughly the same time [36]. In summary, the collision process can be divided into two stages, a first one corresponding to rapid variations of physical quantities, and a second one, characterized by slow variations, in which the target remnant, fairly well equilibrated, loses energy by an evaporationlike process. The transition is not exceedingly sharp, however. We decided to take the stopping time as the time at which the characteristic variation in the second stage is just fully established, indicated for instance by the arrows above the curves in Fig. 2. Admittedly, there is some uncertainty (of 2 to $5 \mathrm{fm} / c$, perhaps) in our procedure, as this criterion points to slightly different times for different observables. We made a systematic investigation of the stopping time for different systems, incident energies, and impact parameters. We finally adopted a smooth function for the variation of $t_{\text {stop }}$ given by

$$
t_{\text {stop }}=f_{\text {stop }} t_{0}\left(\frac{A_{T}}{208}\right)^{0.16},
$$

where $f_{\text {stop }}=1$ and $t_{0}=70 \mathrm{fm} / c$. This time is substantially larger than the values adopted in the standard version (as described in Refs. $[14,36])$, although the criteria are basically the same. This is merely due to the fact that, because of the presence of a diffuse surface, the incident particle is initially situated farther away from the target than in the previous versions. The stopping time is now largely independent of the impact parameter and of the incident energy, for the same reason.

\section{E. Numerical model}

The INC model described above is cast into a numerical code, named INCL4. Compared to the previous version INCL3, it now includes the new ingredients described above and produces the calculated value of $\ell^{*}$ in the output.

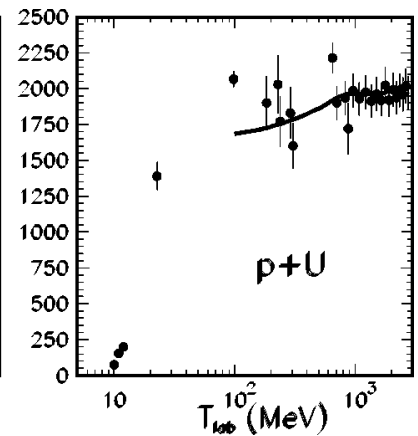

FIG. 3. Total reaction cross sections for proton-induced reactions on three different targets, compared with the predictions of INCL4 (full curves) and INCL3 (dashed curve) models. Experimental data (dots) are from Refs. $[37,38]$.

We stress that there are only two free parameters in the INCL4 version: the potential depth $V_{0}$ and the overall factor $f_{\text {stop }}$ in Eq. (29) governing the stopping time, all other parameters being determined by experiment or previous studies (see Ref. [14] for more information). The arguments of the previous subsection show that the value of $f_{\text {stop }}$ is strongly bound by physical considerations. This is also the case for $V_{0}$, whose value should be taken as the sum of the Fermi energy and the average nucleon separation energy. We are going to present in the next section predictions of the model with the standard values of these parameters, namely, $V_{0}$ $=45 \mathrm{MeV}$ and $f_{\text {stop }}=1$. We will later say a few words on the variation of the results with modifications of these parameters. A meaningful comparison with experimental data requires the introduction of an evaporation model. Here we use the Schmidt evaporation model $[39,40]$, more precisely the version labeled KHSv3p, with always the standard values of the parameters. In the rest of this paper, the INCL4 + KHSv3p ensemble is referred to as "our model" and has thus only two free parameters.

It is often hard to disentangle the respective merits of the INC and the evaporation models. This can possibly be done by comparing results obtained with several evaporation models. In the following we try to concentrate on data that depend as little as possible on evaporation. But, even if it is not our main concern, we will not ignore the question of the dependence upon the evaporation model. We will, however, limit ourselves to a comparison with the results obtained with the Dresner evaporation model [41].

The description of the Schmidt evaporation code can be found in Ref. [40]. It is sufficient for the moment to say that particle $(p, n, \alpha)$ emission is described by the WeisskopfEwing model [42], that the fission model includes a refined fragment mass distribution function [43], and that friction is introduced through a fission delay recipe [44]. We will elaborate a little more on the ingredients in Sec. IV when comparing the two evaporation models.

\section{COMPARISON WITH EXPERIMENTAL DATA}

\section{A. Proton-induced reactions}

\section{Total reaction cross section}

The predictions of our model for this observable are presented in Fig. 3 for three targets and compared with the experimental measurements as well as with the predictions of the previous version of the Liège INC model. One can see 

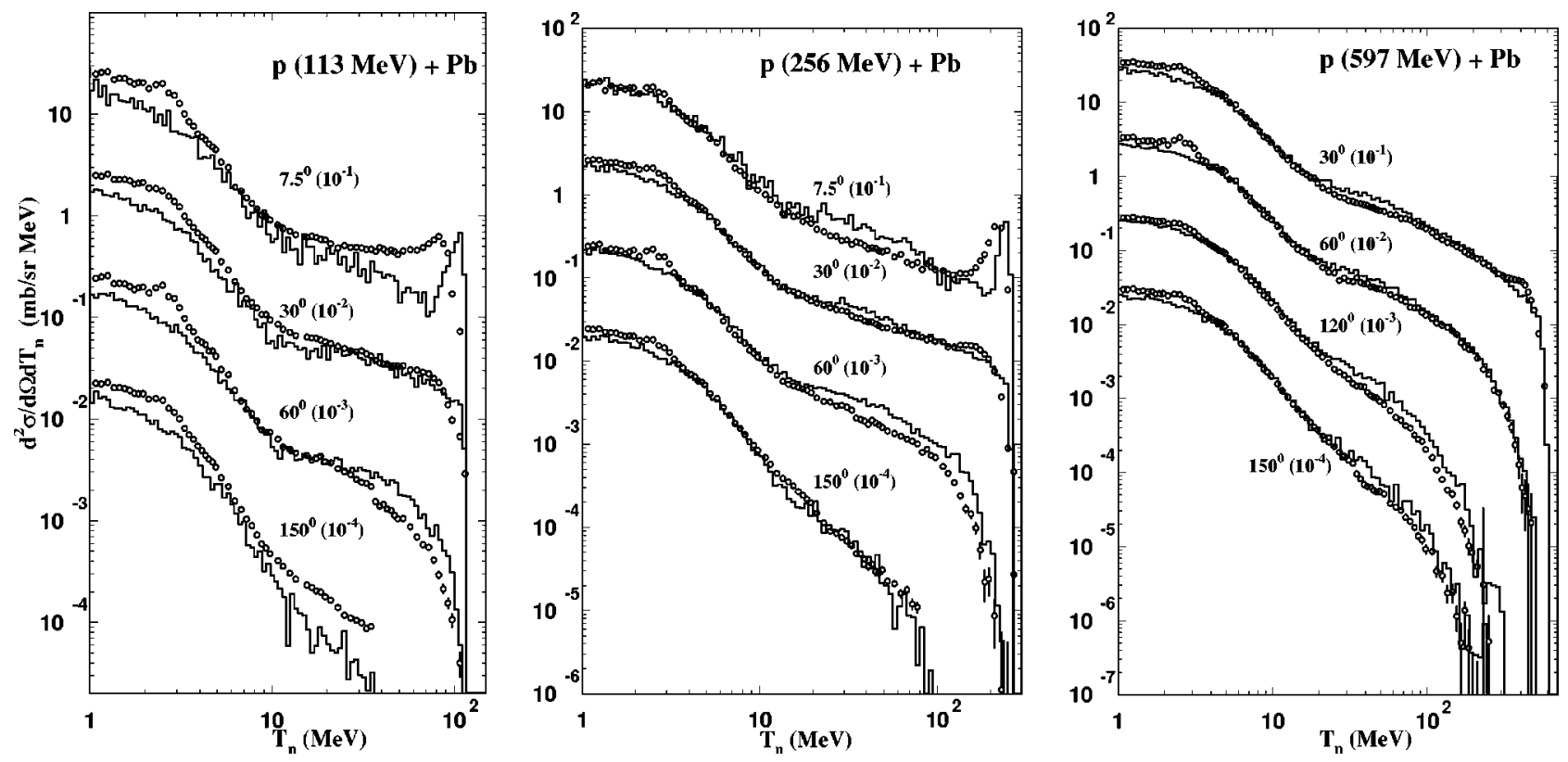

FIG. 4. Neutron double differential cross sections for proton-induced reactions on a $\mathrm{Pb}$ target at $113 \mathrm{MeV}$ (left), $256 \mathrm{MeV}$, (center) and $597 \mathrm{MeV}$ (right) incident energy. Predictions of the INCL4+KHSv3p model are given by the histograms. Data (circles) are from Refs. [45-47]. In order to ease the reading of the figure, the successive spectra have been mutiplied by decreasing powers of 10 , except for $0^{\circ}$, in which case absolute values are given, the multiplying factor being taken as $10^{-1}$ for the first presented nonvanishing detecting angle. The values of the detection angles are given close to the corresponding curves.

that the introduction of a diffuse nuclear surface increases the total cross section: impact parameters larger than the half density radius now contribute. Owing to the large nucleonnucleon cross section, this contribution overcompensates the slight decrease of the contribution of the impact parameters smaller than the half density radius, due to partial transparency. One can see also from Fig. 3 that our INC model gives reasonable values of the total cross section in view of the experimental uncertainties in the whole $100-\mathrm{MeV}-2-\mathrm{GeV}$ incident energy range. One may, however, notice that the cross section is slightly underpredicted below $\sim 200 \mathrm{MeV}$, indicating the inability of the Liège INC model to correctly describe the rise of the total reaction cross section at low incident energy, for light and medium weight targets.

We stress that the total reaction cross section is testing the predictions of the INC model solely. It is totally independent of the evaporation model.

\section{Neutron cross sections}

This is the most important observable for the possible applications to spallation reactions in ADS. We display in Fig. 4 the predictions of our model along with the measurements of double differential neutron production cross sections in proton-induced reactions on $\mathrm{Pb}$ of Refs. [45-47] for 113, 256, and $597 \mathrm{MeV}$, respectively. Figures 5-7 show a similar comparison with the data obtained at SATURNE [19] for the same system at $0.8,1.2$, and $1.6 \mathrm{GeV}$ incident energy, respectively. The targets used in Ref. [19] have some finite thickness: $2 \mathrm{~cm}$ for $\mathrm{Pb}$ and $\mathrm{Th}$, and $3 \mathrm{~cm}$ for $\mathrm{Al}$ and $\mathrm{Zr}$. In view of technological applications, the data are plotted, as

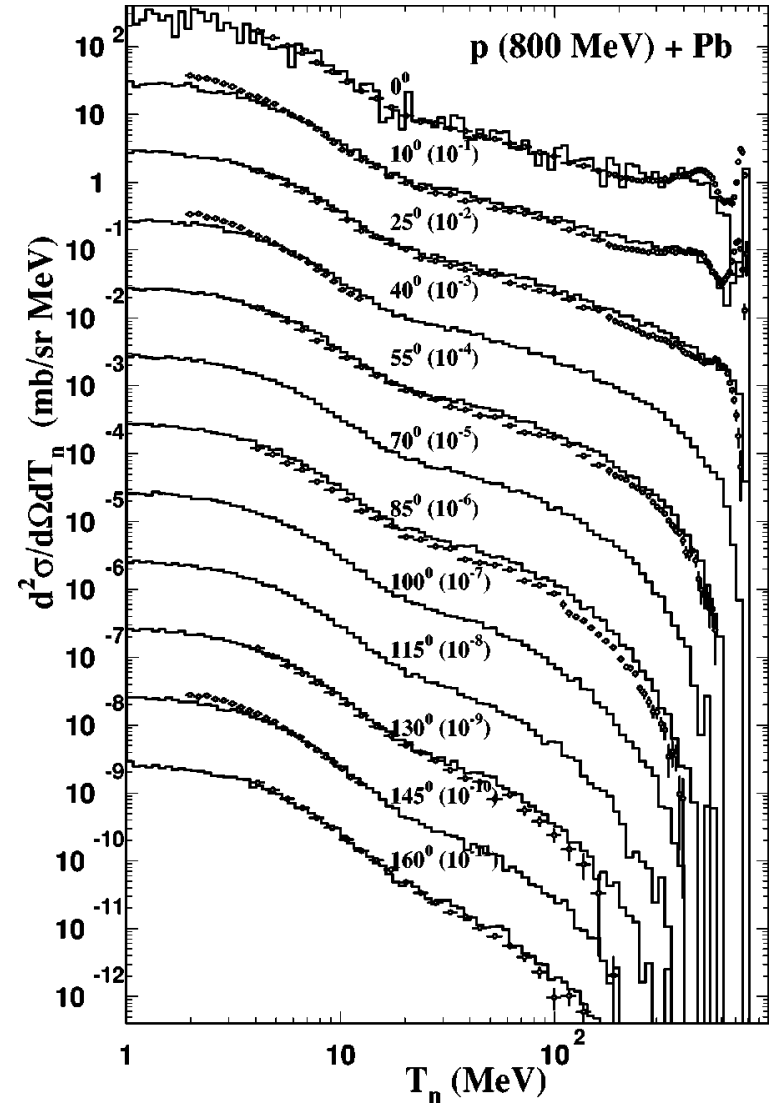

FIG. 5. Same as Fig. 4 at an incident energy of $800 \mathrm{MeV}$. Data (circles) are from Ref. [19]. The value of the target thickness is given in the text. 


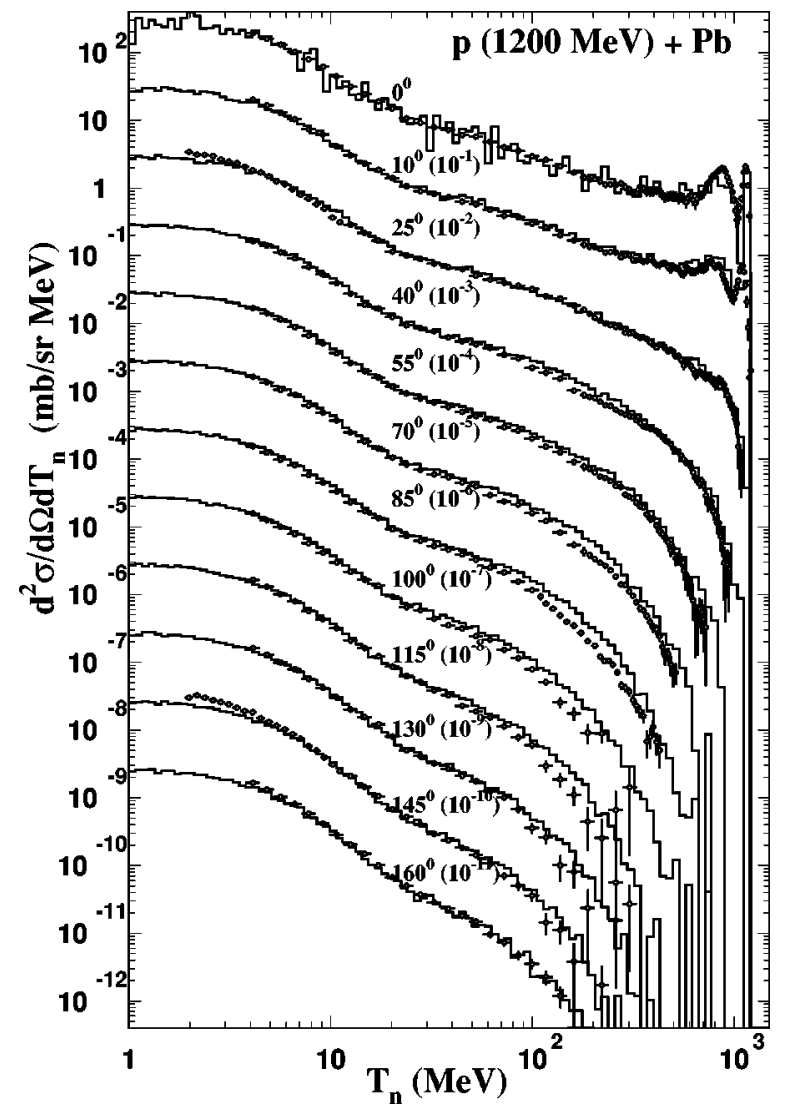

FIG. 6. Same as Fig. 5 for an incident energy of $1200 \mathrm{MeV}$.

traditionally, versus the logarithm of the neutron energy, or the lethargy, since this quantity is a key variable in neutronics.

One can see that the overall agreement is rather good. Although the reader should not be misled by the multiplicative factors introduced in these figures, for the sake of clarity, he can easily realize that the model is able to satisfactorily reproduce the neutron cross section in the whole phase space, i.e., for values extending over five decades. Of course, only the part of the neutron spectra above $\sim 20 \mathrm{MeV}$ is solely due to the INC model. For this component, the values spread over three to four decades only, but such an agreement strongly supports the validity of the cascade approach, i.e., of the multiple-scattering picture. There is a slight tendency for the predictions to be better as the incident energy increases. This may tentatively be ascribed to better and better conditions for the validity of the INC model. Another interesting feature of our new INC model lies in the considerable improvement (see Refs. [14,19] for a comparison) of the prediction for the intensity of the quasielastic peak, i.e., the bump in the neutron energy spectra close to the beam energy at very forward angles. The intensity is correctly reproduced at $0^{\circ}$, but some discrepancy still remains at larger angles. A similar statement can be formulated for the quasi-inelastic peak, due to $\Delta$ excitation, i.e., the peak located at the beam energy minus $\sim 300 \mathrm{MeV}$ at forward angles, though the agreement is less satisfactory. This improvement is, of course, due to the introduction of a diffuse surface, which enhances the probability of having a single charge exchange

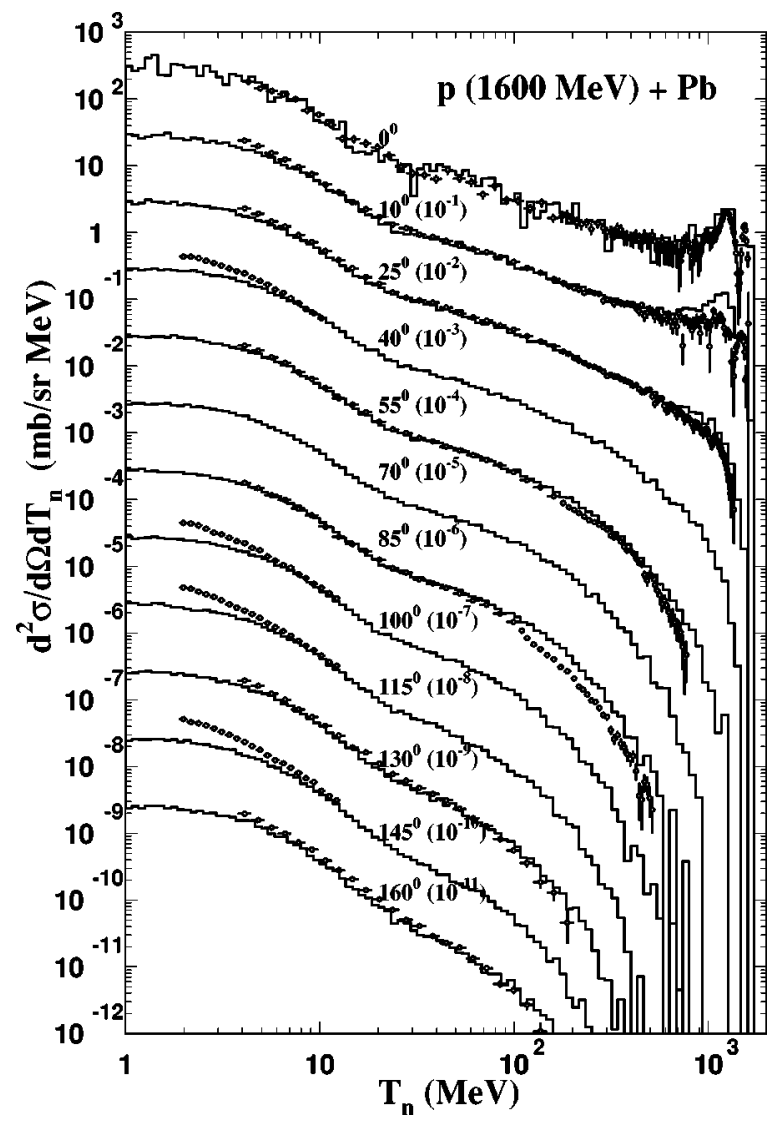

FIG. 7. Same as Fig. 5 for an incident energy of $1600 \mathrm{MeV}$.

(elastic or inelastic) nucleon-nucleon scattering in the forward direction, i.e., a quasifree (elastic or inelastic) $(p, n)$ scattering.

It is also of interest to look at neutron multiplicities, global properties of neutron spectra which are not easily revealed by their inspection. They are given in Table II for $p$-induced reactions on $\mathrm{Pb}$ and sorted on different intervals of neutron energy. One can see that the agreement with our model predictions is fairly good both for the cascade neutrons $(\geqslant 20 \mathrm{MeV})$ and the evaporation neutrons (2-20 $\mathrm{MeV})$. One has to keep in mind that the data do not refer to infinitely thin targets. This slightly distorts neutron spectra. As for the neutron multiplicities, the effect of the target thickness considered here is roughly the following [19]: multiplicities above $20 \mathrm{MeV}$ are reduced by $0.2-0.3$ and multiplicities in the 2-20-MeV interval are basically unchanged. The INCL4 predictions of Table II do not include these corrections. The cascade neutron multipicity is only about onefourth of the total multiplicity, but one should keep in mind that their associated energy flow (multiplicity multiplied by the average kinetic energy) is the major part of the total energy flow $(\sim 80 \%)$, as illustrated in Ref. [19]. In a thick target, these neutrons will generate many more neutrons by further interactions with other nuclei.

A detailed analysis of the comparison between predictions and data reveals, however, some discrepancies. Perhaps the most surprising one is the moderate agreement concerning the location of the quasielastic peak, as illustrated by Fig. 8, which shows a close-up of this region, for intermediate inci- 
TABLE II. Neutron multiplicities, obtained by integration of the experimental double differential cross sections in proton-induced reactions on $\mathrm{Pb}$ nuclei (second column), compared with the predictions of our model (third column) and those from other models. See Sec. VI for more details. Note that ISABEL cannot be run in LAHET above $1 \mathrm{GeV}$.

\begin{tabular}{|c|c|c|c|c|c|c|}
\hline Energy & Expt. & $\begin{array}{c}\text { INCL4 } \\
\text { KHSv3p }\end{array}$ & $\begin{array}{l}\text { TIERCE } \\
\text { Cugnon }\end{array}$ & $\begin{array}{c}\text { LAHET } \\
\text { Bertini }\end{array}$ & $\begin{array}{l}\text { LAHET } \\
\text { ISABEL }\end{array}$ & $\begin{array}{c}\text { LAHET } \\
\text { Bertini-preq }\end{array}$ \\
\hline \multicolumn{7}{|c|}{$\mathrm{Pb} T_{l a b}=800 \mathrm{MeV}$} \\
\hline $0-2 \mathrm{MeV}$ & & 3.3 & 4.9 & 5.61 & 5.13 & 5.37 \\
\hline $2-20 \mathrm{MeV}$ & $6.5 \pm 1.0$ & 6.8 & 6.9 & 8.63 & 6.63 & 7.12 \\
\hline $20 \mathrm{MeV}-E_{\max }$ & $1.9 \pm 0.2$ & 2.5 & 2.2 & 1.75 & 1.92 & 2.13 \\
\hline Total & & 12.5 & 14.0 & 16.0 & 13.7 & 14.4 \\
\hline \multicolumn{7}{|c|}{$\mathrm{Pb} T_{l a b}=1200 \mathrm{MeV}$} \\
\hline $0-2 \mathrm{MeV}$ & & 3.4 & 5.8 & 6.35 & & 6.02 \\
\hline $2-20 \mathrm{MeV}$ & $8.3 \pm 1.0$ & 8.1 & 8.9 & 11.44 & & 9.86 \\
\hline $20 \mathrm{MeV}-E_{\max }$ & $2.7 \pm 0.3$ & 3.1 & 2.8 & 2.45 & & 2.83 \\
\hline Total & & 14.7 & 17.4 & 20.2 & & 18.7 \\
\hline
\end{tabular}

dent energy. The theoretical maximum is situated at too high an energy (by an amount of $\sim 30-40 \mathrm{MeV}$ ). The question of the location and the intensity of the quasielastic peak has been discussed repeatedly in the literature [48-51], mainly at $0^{\circ}$. The downward shift of the peak location that is observed in $(p, n)$ reactions seems to arise from collective effects mixed with the spin dependence of nucleon-nucleon cross section. It is thus natural that we could not correctly reproduce this location. It should be kept in mind, however, that

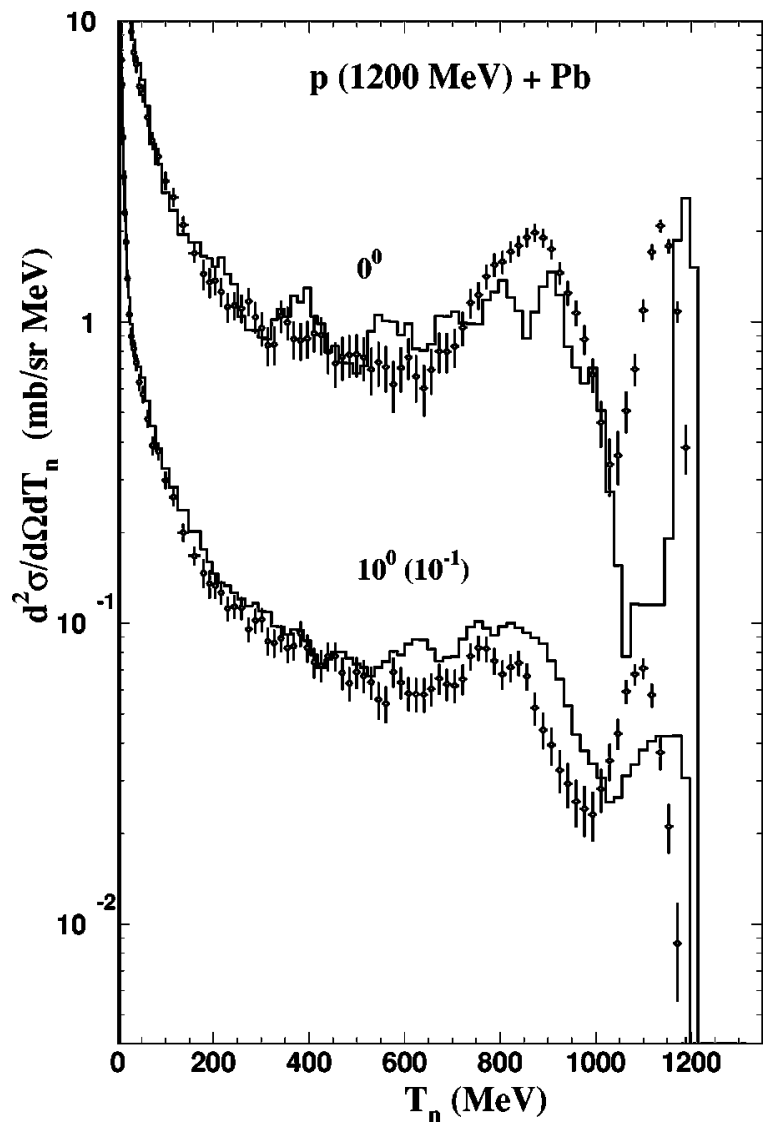

FIG. 8. Same as Fig. 6 with a linear horizontal scale. part of the shift in Figs. 5-7 arises from the thickness of the target [19,21]: about $20 \mathrm{MeV}$ for a 2-cm-thick Pb target. The effect of the target thickness on the neutron spectra amounts to roughly a constant shift of the curves in the semilogarithmic plots shown in Fig. 5 and similar ones, toward low energies. Due to the smooth variation of the curves and the scales, this correction would not be visible on Fig. 5, outside the quasielastic peak.

The so-called quasi-inelastic peak is satisfactorily reproduced in our calculations, as it is illustrated in Figs. 5-7, except at $10^{\circ}$ in $p+\mathrm{Pb}$ reactions at $1.6 \mathrm{GeV}$. In contrast with the quasielastic peak, which can be attributed to a single $(p, n)$ elastic scattering [27], in the quasiinelastic region, the single $(p, n)$ inelastic scattering contribution is superimposed to a background of multiple-scattering contribution. Therefore the agreement for the single inelastic contribution can hardly be estimated. We, however, believe that the quality of our results is partly due to the good parametrization of the $N N \rightarrow N \Delta$ cross section, taken from Ref. [28].

At $800 \mathrm{MeV}$ incident energy and above, the agreement for intermediate neutron energy $(\sim 20-400 \mathrm{MeV})$ is generally better for forward and backward angles than for intermediate angles. At low incident energy, the situation has a tendency to be reversed. It is hard to relate this feature to specific ingredients of our model, as this part of the neutron spectra is coming from multiple scattering. On the other hand, it is remarkable that the multiple scattering component is after all better described than the single scattering components. In our opinion, this indicates that quantum and collective effects are probably fluctuating from one elementary collision to the other and are washed out when the number of collisions rises. Let us also mention that the neutron yield at very backward angles and at low incident energy is underestimated, pointing to a possible lack of pre-equilibrium effects.

The evaporative part of the neutron spectra, i.e., below 20 $\mathrm{MeV}$, is rather well described in our model, except at 113 $\mathrm{MeV}$ incident energy (note that part of the discrepancy is due to our underprediction of the total reaction cross section for this incident energy). This provides with a mixed test of both 


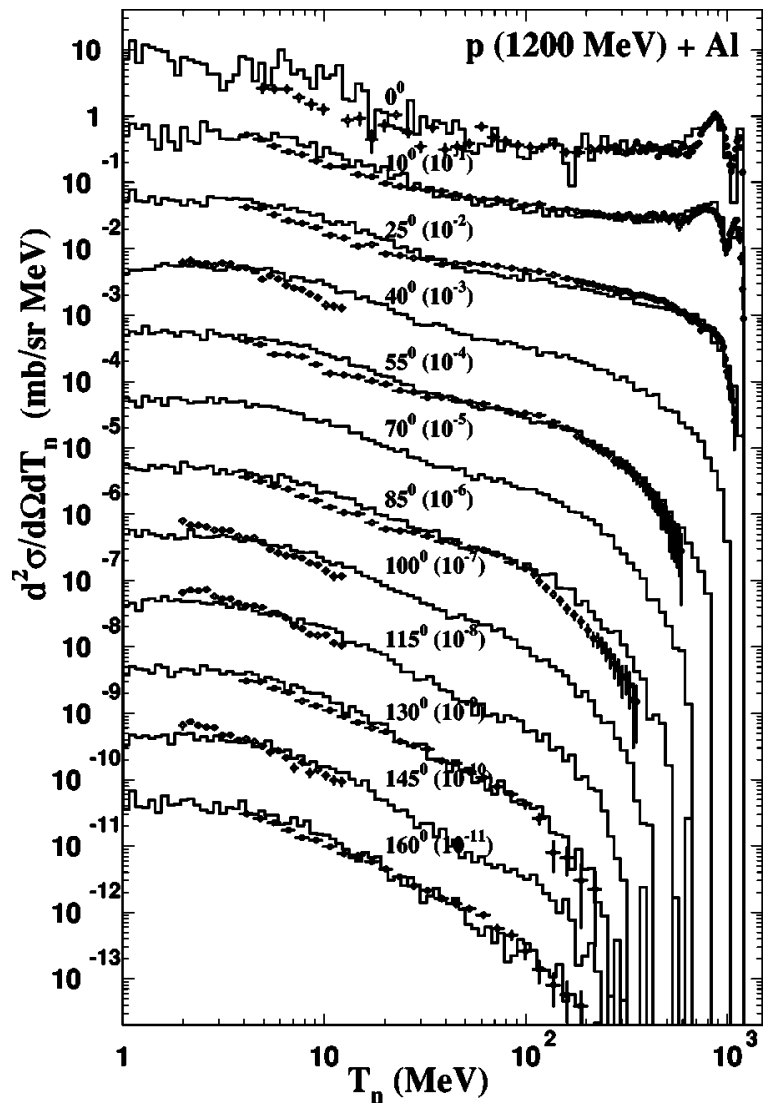

FIG. 9. Same as Fig. 5 for an Al target. Data from Ref. [21].

the INC and the evaporation models: the amplitude, or better the integrated spectrum, is basically related to the number of evaporated neutrons and is largely determined by the excitation energy $E^{*}$ left in the target after the cascade process is over. Indeed, when the evaporation is dominated by neutron emission, the mean number of evaporated neutrons is given, in the Weisskopf-Ewing model, by

$$
\langle n\rangle_{\text {evap }} \approx \frac{\overline{E^{*}}}{S_{n}+2 \bar{T}},
$$

where $S_{n}$ is the mean neutron separation energy and $\bar{T}$ is the mean temperature. Roughly speaking, $\bar{T}=\sqrt{\overline{E^{*} / a}}$, where $a$ is the level density parameter. For average excitation energies considered here, $\bar{T}$ is less than $2-3 \mathrm{MeV}$, and therefore the number of the evaporated neutrons is not very sensitive to the details of the evaporation models, which enter here essentially through the parameter $a$ only. On the other hand, the slopes of the curves are related to the average temperature. The latter depends upon the excitation energy, of course, but it does depend more sensitively upon the leveldensity parameter introduced in the evaporation model than does the number of evaporated neutrons. These considerations agree with the data analysis performed in Ref. [21].

In Figs. 9-11, we investigate the target mass dependence of our results at $1200 \mathrm{MeV}$ incident energy, the results being very similar for other energies and for other targets. One can observe that the cascade parts of the spectra are rather well

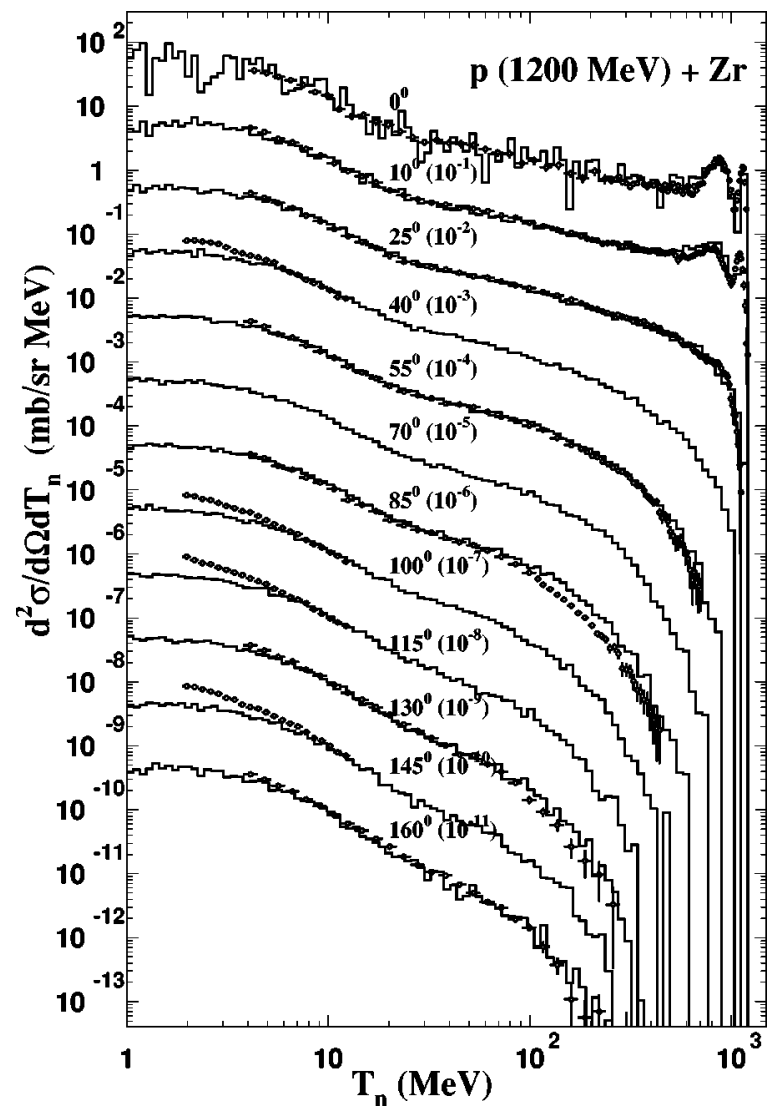

FIG. 10. Same as Fig. 5 for a Zr target. Data from Ref. [21].

reproduced in the whole range of target masses under investigation. The evaporation part of the spectra is also satisfactorily reproduced for large target masses but slightly deteriorates as the mass is decreasing: the predicted slope has a tendency to become too small compared to the data. According to the discussion above, this indicates either a too large predicted excitation energy in the INC model or a too small level density parameter used in the evaporation model.

\section{Proton cross sections}

Reliable experimental data for proton production cross sections are rather scarce. We present in Fig. 12 the comparison of our numerical results with the high precision data of Refs. [52,53], which cover forward angles only. A more thorough investigation of proton data is postponed to a future publication. The agreement is quite good, even in the quasielastic region (small angles). We would like to stress that both the intensity and the location of the peak are now rightly reproduced. For the location, this is in agreement with the discussion of Ref. [51], which indicates that spin and collective effects are much smaller in $(p, p)$ reactions than in $(p, n)$ or other reactions.

\section{Pion production}

This feature is of less importance for ADS applications of spallation reactions, where neutron production is of central interest, although in a thick target produced pions can induce further neutron production. We present in Fig. 13 our results 


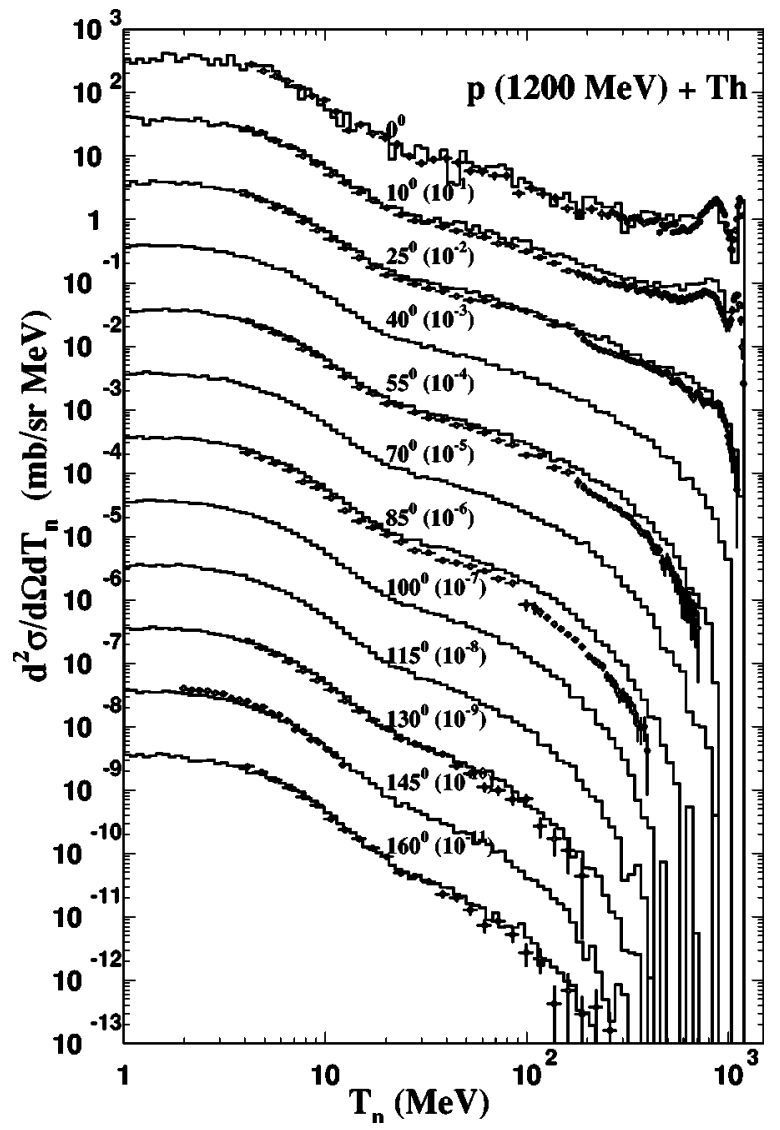

FIG. 11. Same as Fig. 5 for a Th target. Data from Ref. [21].

for the $\pi^{+}$production double differential cross section along with the measurements of Ref. [54]. Although the general trends are reproduced, the total yield is overestimated by a factor of roughly 1.6. The same results (not shown) hold for $\pi^{-}$production. We do not have any clear explanation for this situation, as we use known elementary cross sections and the delta isobar model, which more or less reproduce heavy-ion data. We see two possible origins for the observed discrepancy: the neglect of an average potential for pions or the neglect of medium effects in cross sections and/or decay rates for the processes mentioned in Eq. (1). It has indeed been advocated that medium effects are more important for inelastic nucleon-nucleon collisions than for elastic ones [55].

\section{Other charged-particle production}

We here refer to light composite particles. These particles are produced with quite smaller cross sections, compared to protons, except for ${ }^{4} \mathrm{He}$ particles. Our model can accommodate production of these particles in the evaporation stage only. Experimentally, this is however the main component, the "direct" component being usually much smaller, by one order of magnitude for alpha particles, for instance. The most documented data can be found in Ref. [20]. We just here illustrate the kind of results we get for He particle production in $p+\mathrm{Au}$ collisions at $1.8 \mathrm{GeV}$. The total ${ }^{4} \mathrm{He}$ production cross section is roughly reproduced $(1.43 \mathrm{~b}$ for $1.78 \pm 0.2 \mathrm{~b}$ experimentally), but the ${ }^{3} \mathrm{He}$ emission is neglected in the KHSv3p code $(0.20 \pm 0.08 \mathrm{~b}$ experimentally). On the other hand, the shape of the spectrum is not correctly reproduced, as shown in Fig. 14 (which excludes emission at polar angles between $66^{\circ}$ and $114^{\circ}$, as in the experiment). As explained in Refs. [20,56], this may come from (i) either an incorrect excitation energy distribution after INC, (ii) or the Coulomb barrier which is unadequately chosen. We are inclined to believe that the second reason is the most important. This is supported by the results shown in Fig. 14 and obtained with another evaporation model, the Dresner one (see discussion below). This time the barriers are smaller, perhaps a little bit
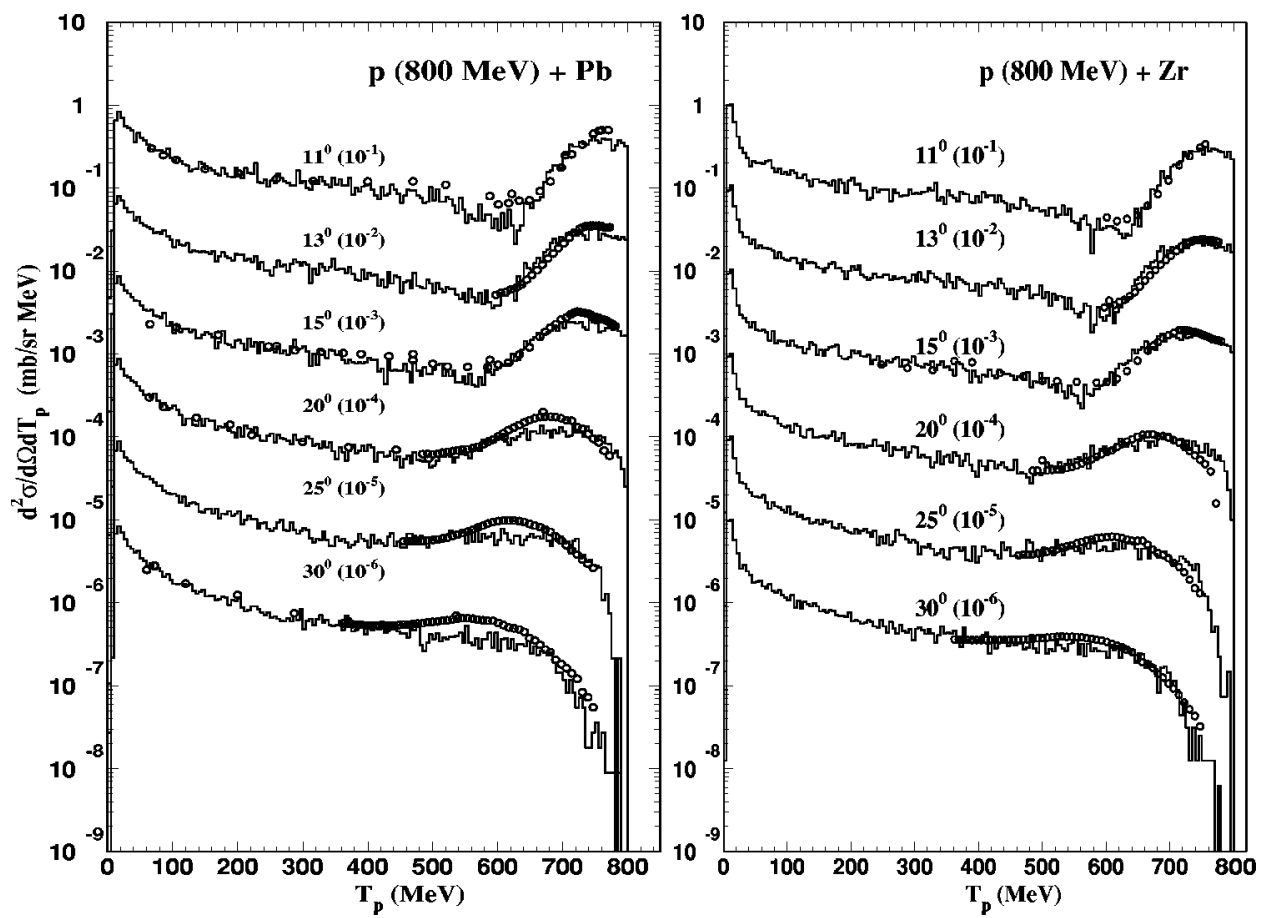

FIG. 12. Proton double differential cross sections for protoninduced reactions on a $\mathrm{Pb}$ target (left panel) and on a $\mathrm{Zr}$ target (right panel) at $800 \mathrm{MeV}$ incident energy. The predictions of the INCL4+KHSv3p model are given by the histograms. Data (circles) are from Refs. [52,53]. 


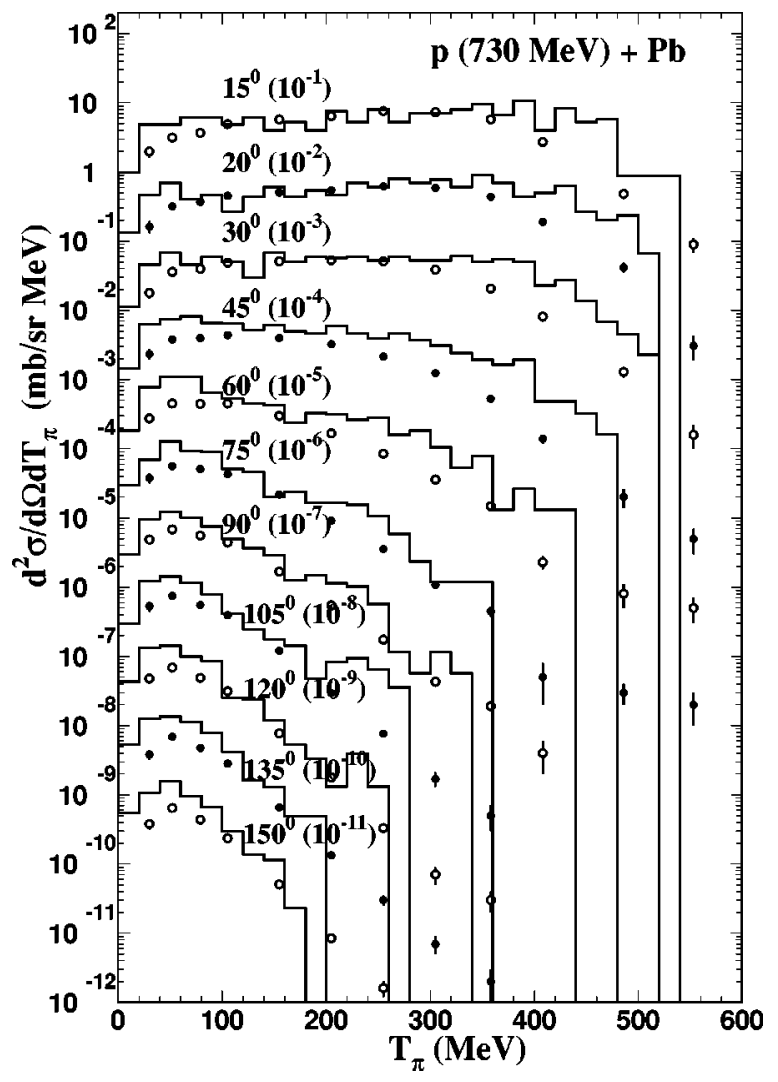

FIG. 13. Positive pion double differential cross sections for proton-induced reactions on a $\mathrm{Pb}$ target at $730 \mathrm{MeV}$ incident energy. The predictions of the INCL4+KHSv3p model are given by the histograms. Data (circles) are from Ref. [54].

too small. In the two cases, the calculations fail to reproduce the tail of the spectrum, above $\sim 40 \mathrm{MeV}$, which arises from nonevaporative emission of particles. Let us also mention that the previous version of the Liège INC model, associated with the GEMINI evaporation code, yields much better results [20], for the evaporation component.

From Table III, it can also be seen that our model satisfactorily reproduces the proton multiplicities, within the experimental cuts $(2-26 \mathrm{MeV}, 2-49 \mathrm{MeV}, 2-76 \mathrm{MeV}$ for $p, d$, and $t$, respectively). It fails, however, to reproduce the total $H$ multiplicity, because it does not include $d$ and $t$ evaporation.

\section{Residue production}

We limit ourselves to recent data obtained in so-called inverse-kinematics experiments, which consists of bombarding a $\mathrm{H}$ target with a heavy projectile and using a fragment mass separator in the forward direction. This provides a direct access to the residue production yields, just after the evaporation stage and before the $\beta$ decays (except for the very few residues which have a very small period), in contrast with standard radiochemical and $\gamma$-spectroscopy methods [57-59]. The latter are, however, very useful as they can readily provide excitation functions.

Typical results are shown in Figs. 15 and 16 along with the data of Ref. [60] for the ${ }^{208} \mathrm{~Pb}+p$ reaction at $1 \mathrm{GeV}$ per

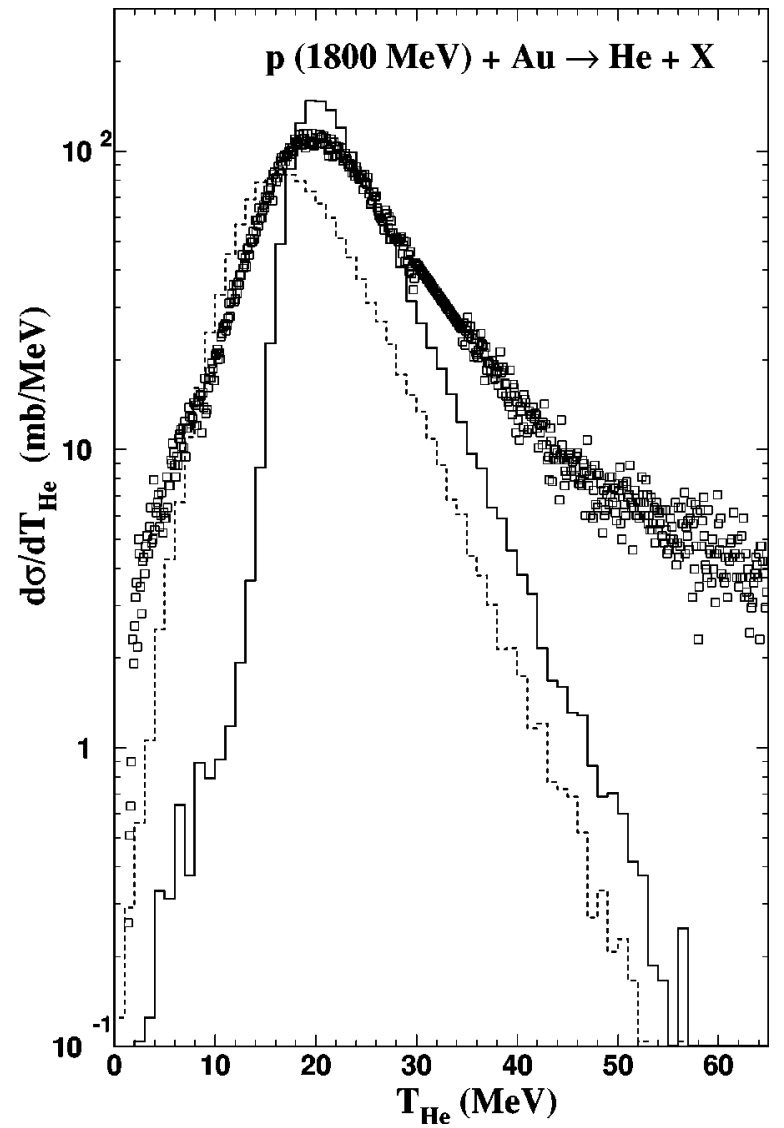

FIG. 14. He particle energy differential cross section for protoninduced reactions on a $\mathrm{Au}$ target at $1.8 \mathrm{GeV}$ incident energy. The predictions of the INCL4+KHSv3p model are given by the solid histogram. The dashed histogram corresponds to substituting the Dresner model to the KHSv3p evaporation model. Data (squares) are from Ref. [20]. See text for details.

nucleon. A good agreement is achieved both for charge and mass spectra. They exhibit two components: a so-called fragmentation peak, collecting the heavy residues left after evaporation of the remnant, and a broad fission peak centered around $A \sim 90$. Compared to the previous version of our INC model [14,23,61], the yields for isobars and isotopes close to the target are now correctly reproduced. This is a direct consequence of the introduction of a diffuse surface, which enhances the rate of events with small excitation energy. Our results underestimate the yield in the low-mass (and low-charge) side of the fragmentation peak. These isotopes are generated mostly in the highest excitation energy events. Our numerical results seem to indicate that the excitation energy distribution is not broad enough. We will come back to this point.

It is also remarkable that the fission peak is very nicely described. This is a strong point of the Schmidt evaporationfission model [40]. The shape of this peak is basically given by the fission fragment mass distribution function introduced in this model (see Sec. IV for some remarks). The height of the peak results from the evaporation-fission competition: it is sensitive to the fission delay, but also to the remnant angular momentum $\ell^{*}$ distribution generated by the INC. Replacing the latter by the de Jong prescription [62] for $\ell^{*}$ 
TABLE III. Neutron and light charged particle multiplicities in proton-induced reactions, as predicted by our INC model used with two evaporation models (Dresner [41] and KHSv3p [40]). For the gold target case, the predictions are compared with the data of Ref. [20].

\begin{tabular}{|c|c|c|c|c|c|}
\hline Particle multiplicity & \multicolumn{2}{|c|}{ INCL4+ Dresner } & \multicolumn{2}{|c|}{ INCL4+ KHSv3p } & \\
\hline \multicolumn{6}{|c|}{$p(1.2 \mathrm{GeV})+\mathrm{Pb}$} \\
\hline$n E<2 \mathrm{MeV}$ & & & & 3.41 & \\
\hline$n 2<E<20 \mathrm{MeV}$ & & & & 8.12 & \\
\hline$n E>20 \mathrm{MeV}$ & & & & 3.17 & \\
\hline$p E<20 \mathrm{MeV}$ & & & & 0.70 & \\
\hline$p E>20 \mathrm{MeV}$ & & & & 2.53 & \\
\hline$d$ & & & & & \\
\hline$t$ & & & & & \\
\hline${ }^{3} \mathrm{He}$ & & & & & \\
\hline$\alpha$ & & & & 0.61 & \\
\hline \multicolumn{6}{|c|}{$p(1.8 \mathrm{GeV})+\mathrm{Au}$} \\
\hline & no cut & cut & no cut & cut & experiment [20] \\
\hline$n E<2 \mathrm{MeV}$ & 3.79 & & 3.07 & & \\
\hline$n 2<E<20 \mathrm{MeV}$ & 7.35 & & 8.15 & & \\
\hline$n E>20 \mathrm{MeV}$ & 3.63 & & 3.71 & & \\
\hline$n$ & 14.77 & & 14.93 & & \\
\hline$p E<20 \mathrm{MeV}$ & 1.59 & & 1.07 & & \\
\hline$p E>20 \mathrm{MeV}$ & 2.89 & & 3.00 & & \\
\hline$p$ & 4.48 & 1.84 & 4.07 & 1.40 & $1.20 \pm 0.12$ \\
\hline$d$ & 0.53 & 0.52 & & & $0.60 \pm 0.12$ \\
\hline$t$ & 0.29 & 0.29 & & & $0.35 \pm 0.08$ \\
\hline $\mathrm{H}$ & 5.30 & 2.65 & 4.07 & 1.40 & $2.15 \pm 0.32$ \\
\hline${ }^{3} \mathrm{He}$ & 0.017 & 0.017 & & & $0.12 \pm 0.05$ \\
\hline$\alpha$ & 0.69 & 0.69 & 0.83 & 0.83 & $1.03 \pm 0.12$ \\
\hline $\mathrm{He}$ & 0.72 & 0.72 & & & $1.15 \pm 0.17$ \\
\hline
\end{tabular}

(inspired from the ablation-abrasion model for heavy-ion collisions) reduces the fission yield by roughly a factor 2 , as demonstrated by Fig. 15. For the particular case under consideration, $\left\langle\ell^{*}\right\rangle \approx 16$ in our results, compared to $\left\langle\ell^{*}\right\rangle \approx 8.7$ for the de Jong prescription. Our results lead us to believe that the friction parameter used in the KHSv3p model is given an adequate value. Let us recall this value: $\beta=1.5$ $\times 10^{21} \mathrm{~s}^{-1}$, corresponding to a fission delay of approximately $2 \times 10^{-21} \mathrm{~s}[22]$.

The isotopic distributions (for the most abundantly produced isotopes in the fragmentation and fission peaks) are displayed in Fig. 16. There is an overall good agreement, like for the integrated distributions. It is remarkable that the agreement is almost perfect for the fission isotopes. This stems from the Schmidt model for the mass partitioning in fission, but also from the correct charge-mass distribution of the fissioning remnants, which results from both the INC and evaporation models. Predicted heavy-isotope (from $\mathrm{W}$ to $\mathrm{Pb}$ ) distributions present small but definite discrepancies: the yield is slightly overestimated on the extreme heavy mass side and the detail of the peak of the $\mathrm{Tl}$ and $\mathrm{Pb}$ distributions is not reproduced. This last feature is to be attributed mainly to the INC, as these residues are produced by the removal of a very small number of nucleons. This is in keeping with the difficulty of reproducing correctly the one-collision part in neutron cross sections. We will come back to this point

The experimental data for the $\mathrm{Au}+p$ reaction at $800 \mathrm{MeV}$ per nucleon $[63,64]$ are shown in Figs. 17 and 18, along with our predictions. The same kind of agreement with the same strong points and the same deficiencies as for the $\mathrm{Pb}+p$ case is to be noticed. Let us remark, however, that the predicted fission peak seems to be shifted by two or three units toward higher values of $Z$ (or $A$ ) in the calculation. This time, the isotope yield for the target charge plus one unit has been measured (the last point in the $Z$ distribution) and is correctly reproduced. The production of these isotopes is due to $(p, x n)$ reactions or, to a lesser extent, to $\left(p, p \pi^{-} x n\right)$, $\left(p, \pi^{0} x n\right)$, e.g., reactions. Let us also mention that residues with mass number $A$ larger or equal to $A_{T}$ have not been measured. Our calculations predict a cross section of $10 \mathrm{mb}$ for $A=A_{T}$ and upper bound of $6 \mu \mathrm{b}$ for $A=A_{T}+1$. The latter case presumably does not correspond to complete fusion, but to the $\left(p, \pi^{0}\right)$ reaction, that implies a smaller, though still sizable, energy-momentum transfer.

Figure 18 shows that the isotopic distributions for the fragmentation peak region are satisfactorily reproduced. Those for the fission peak (not shown) are reproduced with a similar accuracy.

We display in Fig. 19 the results for ${ }^{56} \mathrm{Fe}$ induced reactions on hydrogen at $573 \mathrm{MeV}$ per nucleon [65]. One can 

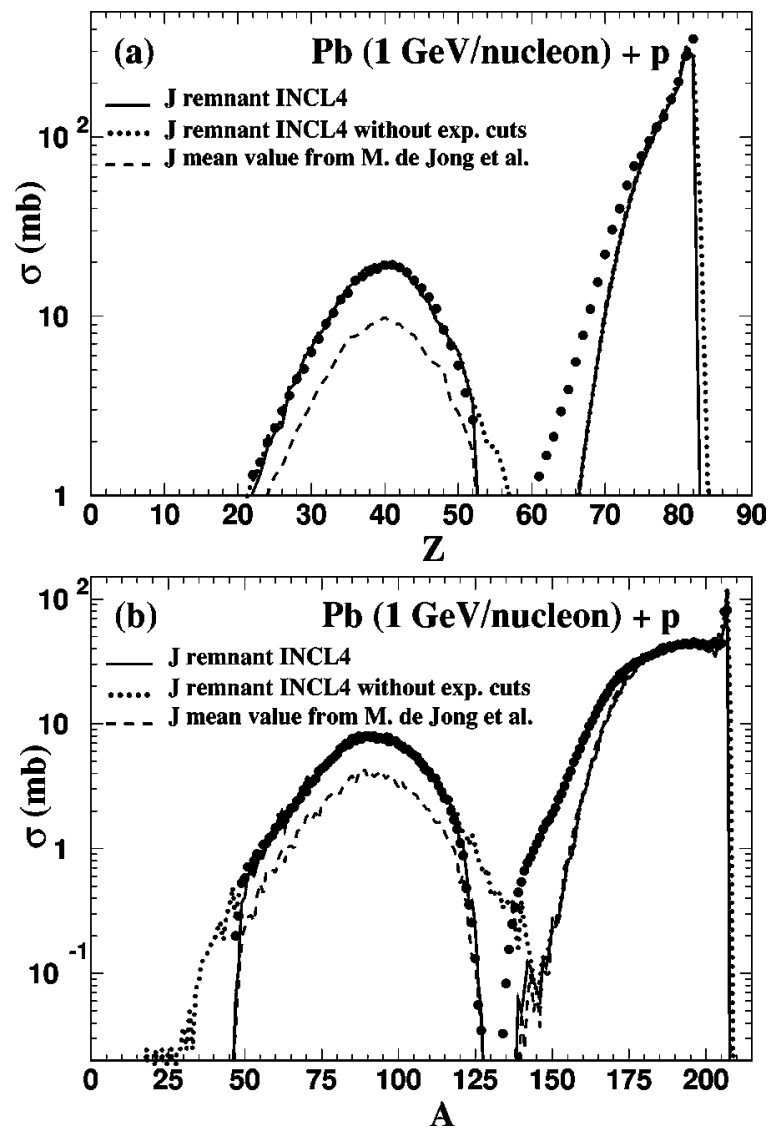

FIG. 15. Residue charge (upper part) and mass (lower part) production cross sections for ${ }^{208} \mathrm{~Pb}$-induced reactions on a $\mathrm{H}$ target at $1.0 \mathrm{GeV}$ per nucleon incident energy. The predictions of the INCL4+ KHSv3p model are given by the lines, including all isotopes (dotted lines) or only those which have been measured experimentally (full lines). The dashed lines display the results obtained when the de Jong prescription is used for the remnant angular momentum. Data (dots) are from Ref. [60].

observe that the most important yields are satisfactorily reproduced, but that the predictions present the same shortcoming as before for residues far from the target. In addition, there is an experimental odd-even effect superimposed on the increasing background, which is not accounted for in the calculations. This perhaps points to some deficiency of the Schmidt evaporation model, which has been tested on heavy nuclei mostly. We would like to emphasize that our model predicts a substantial cross section $(6 \mathrm{mb})$ for $Z=Z_{T}+1$, which can be reached by $(p, n)$ or $(p, \pi)$ reactions only. This figure is to be compared with a lower bound of $\sim 2 \mathrm{mb}$, quoted in Ref. [66].

The isotopic distributions for the same reaction are given in Fig. 20 and compared with our predictions. The latter are rather good for heavy isotopes. The agreement gets less and less good as the charge of the isotopes decreases, in keeping with Fig. 19, pointing to an unsatisfactorily proton-neutron competition in the Schmidt evaporation model for light nuclei.

\section{Recoil of the residues}

We compare our predictions for this quantity with the measurements of Ref. [60]. The latter are based on inverse- kinematics experiments: the proton is at rest in the lab system and the remnant flies away with a large velocity at very forward angles. Its tranverse momentum is very small compared to the longitudinal one. In Ref. [60], only the total momentum is measured and it is identified confidently with the longitudinal momentum. The average longitudinal velocity is presented in Fig. 21, after transformation into the incident $\mathrm{Pb}$ rest frame (they are then negative, in contrast with the usual direct kinematics case), as a function of mass loss $\Delta A\left(=A_{T}-A\right)$. As expected, the absolute value is increasing with mass loss. Indeed, in the cascade stage, the average mass loss, the average excitation energy and the average momentum transfer are increasing with the number of collisions. They are roughly proportional to each other. Evaporation is not contributing very much to the average recoil velocity. It just contributes to a linear rescaling of the mass loss. The first panel of Fig. 21 shows the comparison of the experimental average recoil energies with our predictions, evaluated in the same way as in the experiment, i.e., calculated in the $\mathrm{H}$ rest frame and transformed back in the $\mathrm{Pb}$ rest frame, using the same assumption as above. We checked in fact that the direct calculation in the $\mathrm{Pb}$ rest frame avoiding this assumption gives basically the same result, justifying in some sense the procedure of Ref. [60]. Our model reproduces the general trend with an overall shift towards larger (in absolute value) recoil velocity. We have no simple interpretation of this shift. One has, however, to realize that it is very unlikely that the removal of one or two nucleons occurs with no recoil. Let us finally remark that the erratic behavior of the theoretical recoil velocity for large mass losses is simply coming from the poor statistics of the calculation for these isotopes (see Fig. 15), and has thus no real significance. This remark applies also to the other panels of Fig. 21.

The rms fluctuation of the longitudinal velocity $\sigma_{\beta_{\|}}$is well described by our model (see second panel of Fig. 21). In the INC model, fluctuations arise from the fluctuating momentum transfer in individual collisions. There is no correlation between fluctuations in successive collisions. Therefore the total momentum transfer should roughly display random-walk characteristics: the variance should be proportional to the average momentum loss, which is also basically proportional to the mass loss. Evaporation is not contributing to the average drift, as we already said, but adds further fluctuations. The latter are, however, less important than those brought by the cascade stage, because the evaporation process is much softer than the INC process. Therefore for mass losses which do not involve a tremendous evaporation process, let us say for mass loss $\lesssim 40$, the fluctuations are presumably largely coming from the cascade stage. One indeed observes in Fig. 21 that the experimental variance $\left(\sigma_{\beta_{\|}}\right)^{2}$ is roughly proportional to the mass loss. In our opinion, this feature is very important: it is the clear fingerprint of a random-walk process and provides a strong support of the basic feature of the INC model: the separation or independence of the successive random collisions.

In Ref. [60], the average recoil energy is evaluated, in spite of the fact that the perpendicular velocity is not measured. This is done by assuming that the average transverse 


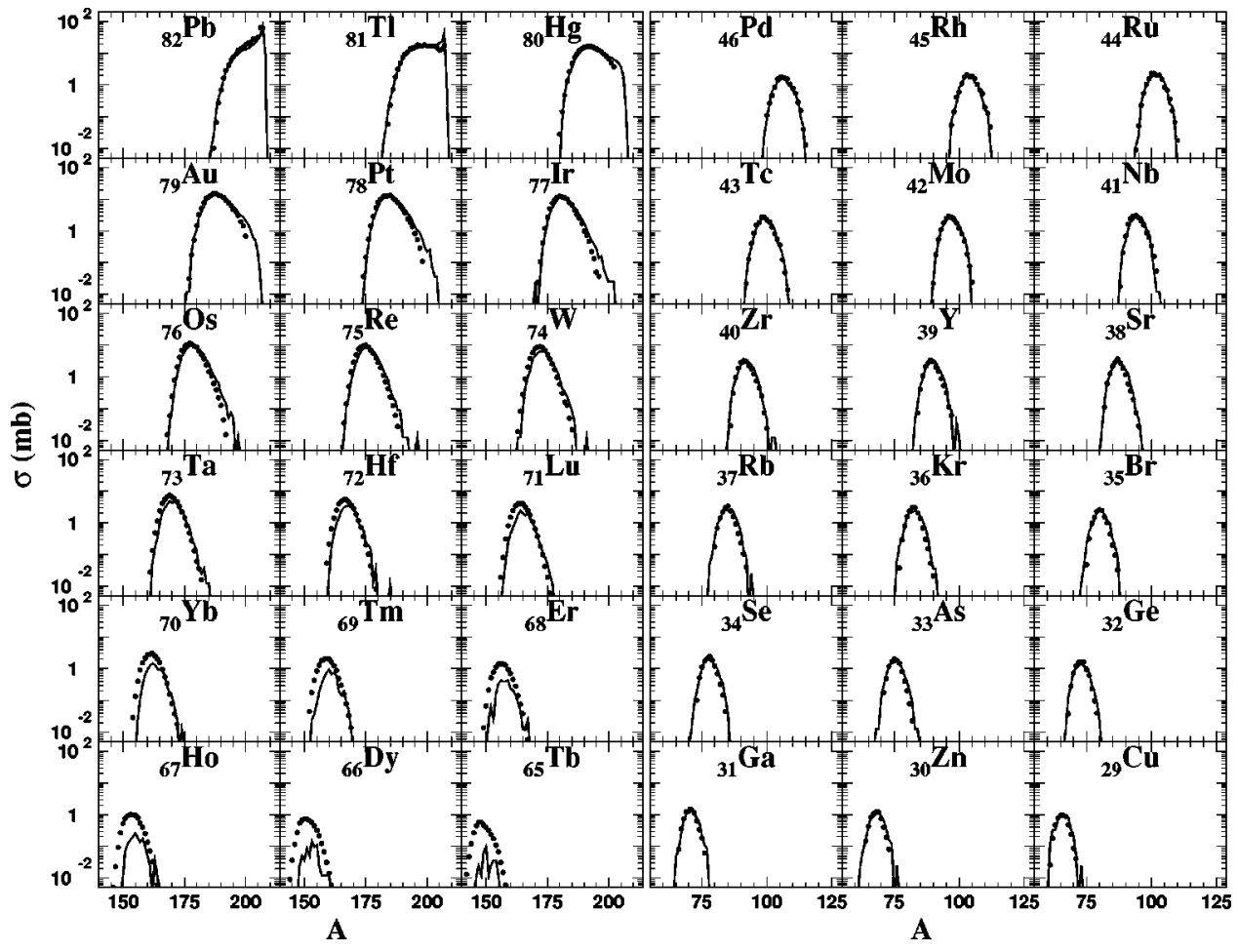

FIG. 16. Isotopic distributions of the nuclides produced in ${ }^{208} \mathrm{~Pb}$-induced reactions on a $\mathrm{H}$ target at $1.0 \mathrm{GeV}$ per nucleon incident energy. Only the most representative isotopes are shown. The predictions of the INCL4 + KHSv3p model are given by the full lines. The numbers near the chemical symbols give the charge number. Data (dots) are from Ref. [60].
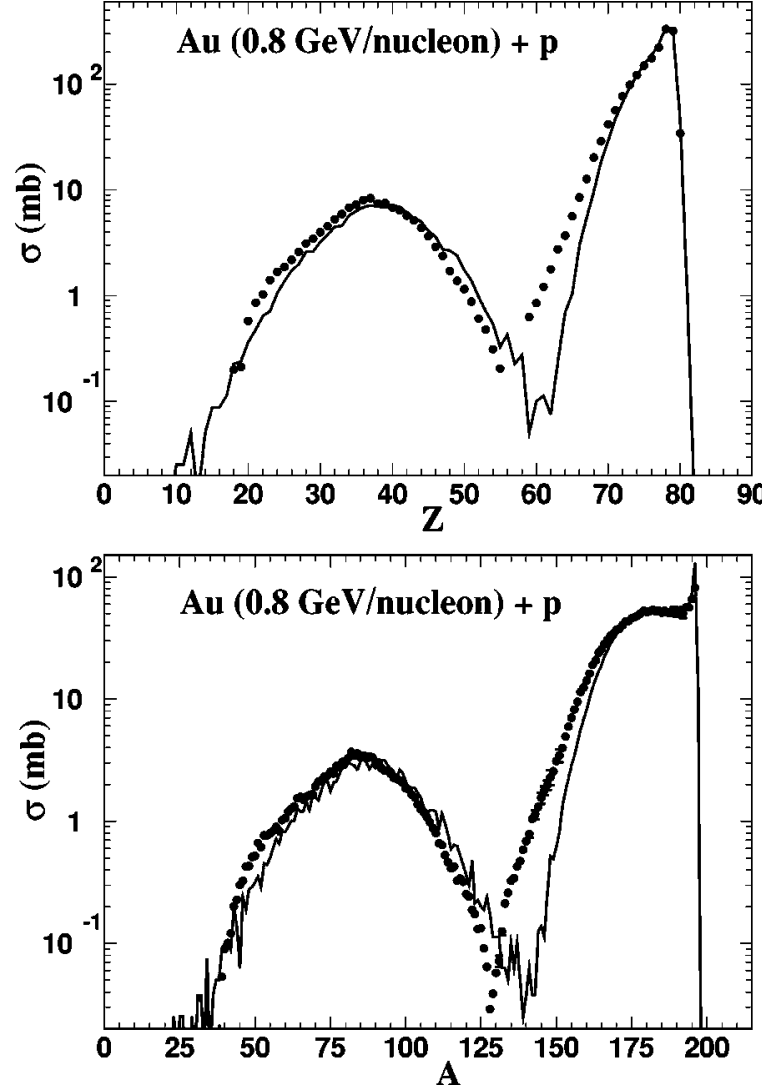

FIG. 17. Residue charge (upper part) and mass (lower part) production cross sections for ${ }^{197} \mathrm{Au}$-induced reactions on a $\mathrm{H}$ target at $0.8 \mathrm{GeV}$ per nucleon incident energy. Predictions of the INCL4 $+\mathrm{KHSv} 3 \mathrm{p}$ model are given by the full lines. Data (dots) are from Refs. [63,64].

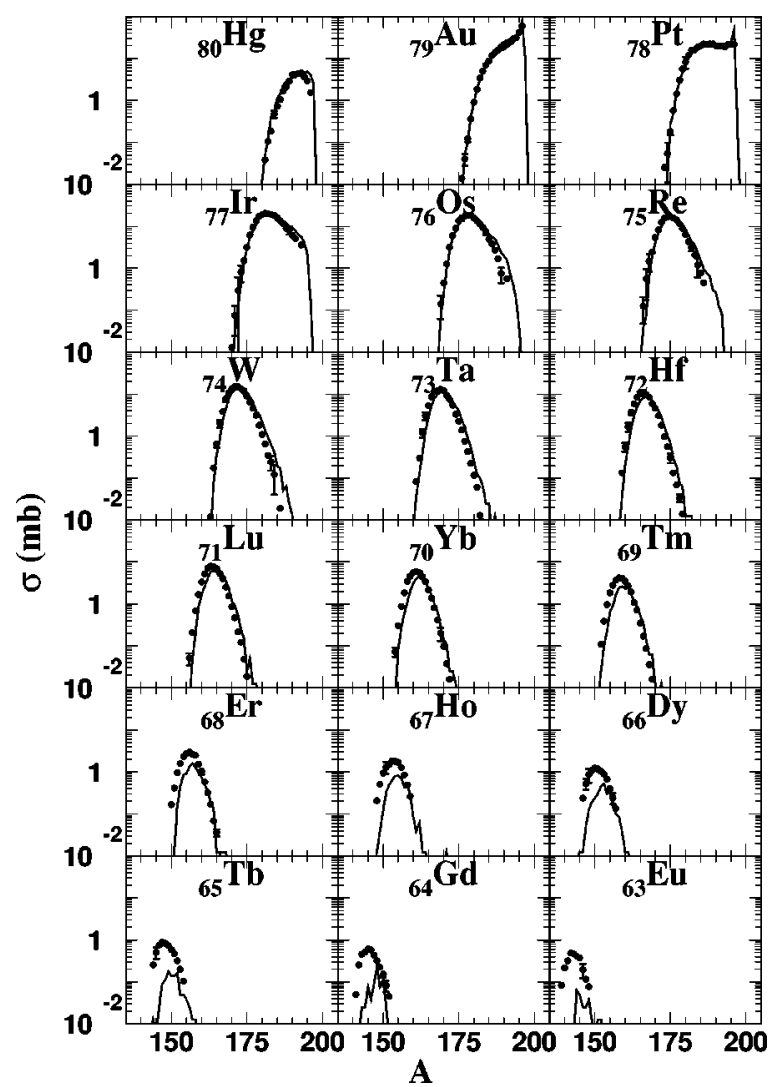

FIG. 18. Isotopic distributions of the nuclides produced in ${ }^{197} \mathrm{Au}$-induced reactions on a $\mathrm{H}$ target at $0.8 \mathrm{GeV}$ per nucleon incident energy. Only the fragmentation peak is considered here. Predictions of the INCL4 + KHSv3p model are given by the full lines. Data (dots) are from Ref. [63]. 


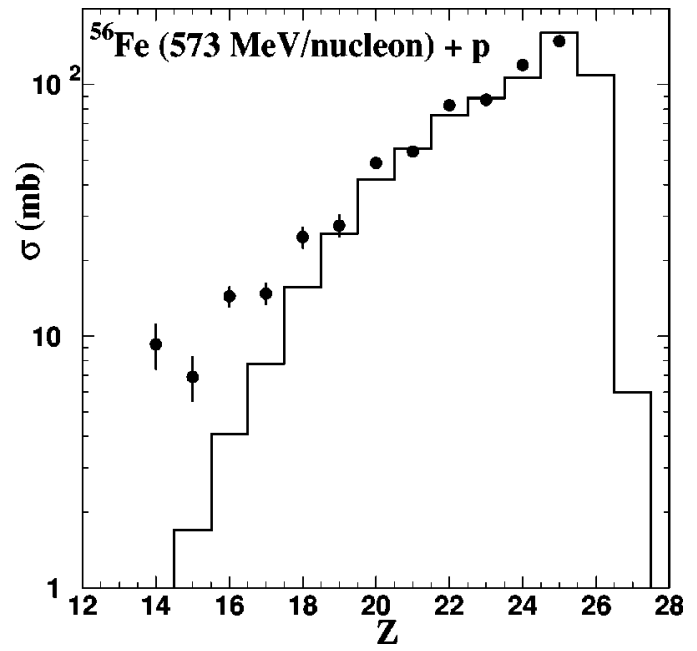

FIG. 19. Residue charge production cross sections for ${ }^{56} \mathrm{Fe}$-induced reactions on a $\mathrm{H}$ target at $573 \mathrm{MeV}$ per nucleon incident energy. Predictions of the INCL4 + KHSv3p model are given by the histogram. Data (dots) are from Ref. [65].

velocity vanishes and that fluctuations of the recoil velocity are the same in the three directions. One so gets, in the nonrelativistic formulation,

$$
\left\langle E_{r e c}\right\rangle=\frac{1}{2} A_{r e m} m_{N} c^{2}\left[\left\langle\beta_{\|}\right\rangle^{2}+3\left(\sigma_{\beta_{\|}}\right)^{2}\right]
$$

The comparison between our theoretical values for the recoil energy and the experimentally reconstructed ones is given in the lower panel of Fig. 21. We display two sets of theoretical results: the values of the recoil energy constructed from our calculations with the same procedure as just discussed above, with the same assumption (squares), and the values taken directly from our calculation (triangles). The two sets nearly coincide with each other, supporting once again the assumption made by the authors of Ref. [60], except for the large mass losses, where the statistics are not good enough. The agreement between theory and experiment is rather nice. For very low mass loss, there is a slight discrepancy, due to the one observed for the average longitudinal velocity, which influences the recoil energy in this mass loss region only, where the recoil energies are small anyway. For large mass loss, the value of the average recoil energy is basically coming from the fluctuations of the velocity, which are well described by our model, as explained above. Let us finally notice that the fluctuations of the recoil energy, not accessed by the experimental procedure of Ref. [60], appear to be rather large in our calculations, about two-thirds of the average value.

\section{B. Deuteron induced reactions \\ 1. Neutron cross sections}

We investigated within our model the properties of the double differential neutron production cross sections and compared with the data on deuteron-induced reactions obtained at SATURNE [67] for 0.8 and $1.6 \mathrm{GeV}$ incident energy and different targets, respectively. Our results and the data in their final shape will be published in the near future. We nevertheless can announce that the agreement is qualitatively the same as for proton-induced reactions. We just here want to draw the attention to two specific features. The first one is illustrated by Fig. 22, which shows that some neutrons are emitted with a velocity larger than the beam velocity. The bump in the neutron spectra is due to the deuteron breakup: the proton inside the deuteron interacts strongly whereas the neutron flies away practically undisturbed. When the incident neutron has a velocity in the forward direction with respect to the deuteron c.m. motion, the latter is added to the incident velocity, when the neutron is freed during the breakup. In reality, there is a slight interaction (not included in our approach) that puts it on the mass shell with the same momentum. The fact that our calculation can account quantitatively for this phenomenon means that the momentum distribution of the neutron inside the deuteron is more important than the way the little extra energy is provided. This is also supported by the fact that it is essential to use a realistic wave function, here based on the Paris potential, to reproduce the data at $10^{\circ}$. In particular, using a Gaussian momentum distribution for the deuteron (with the parameters of Table I) fails to reproduce the tail of the neutron spectrum, as shown by Fig. 22.

The second point deals with the predicted nucleon multiplicities (see Table IV). They are larger in deuteron-induced

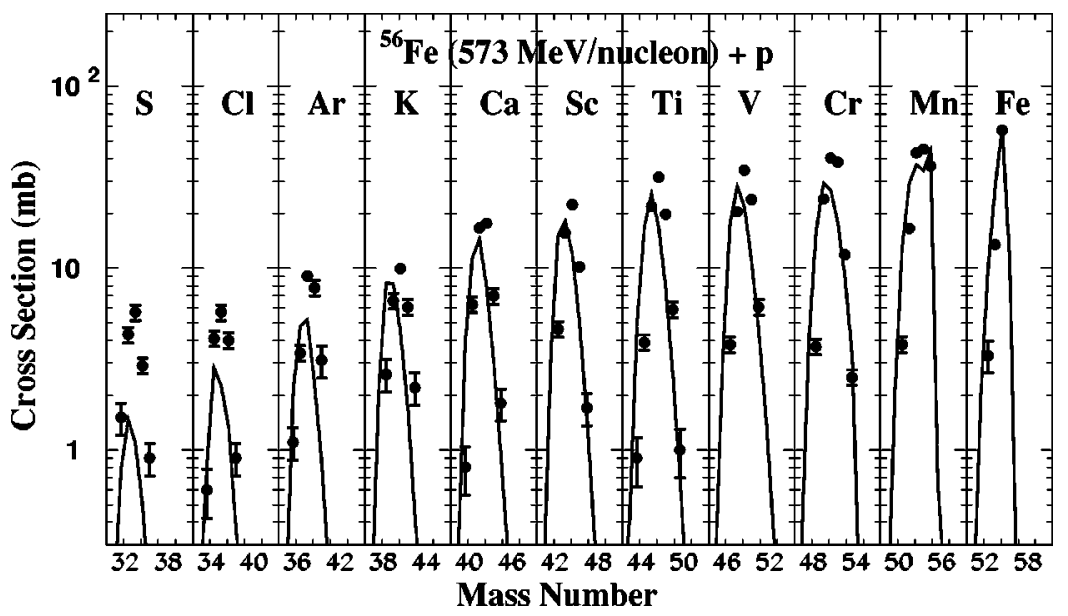

FIG. 20. Isotopic distributions for ${ }^{56} \mathrm{Fe}$-induced reactions on a $\mathrm{H}$ target at $573 \mathrm{MeV}$ per nucleon incident energy. Predictions of the INCL4 + KHSv3p model are given by the full lines. Data (dots) are from Ref. [65]. 

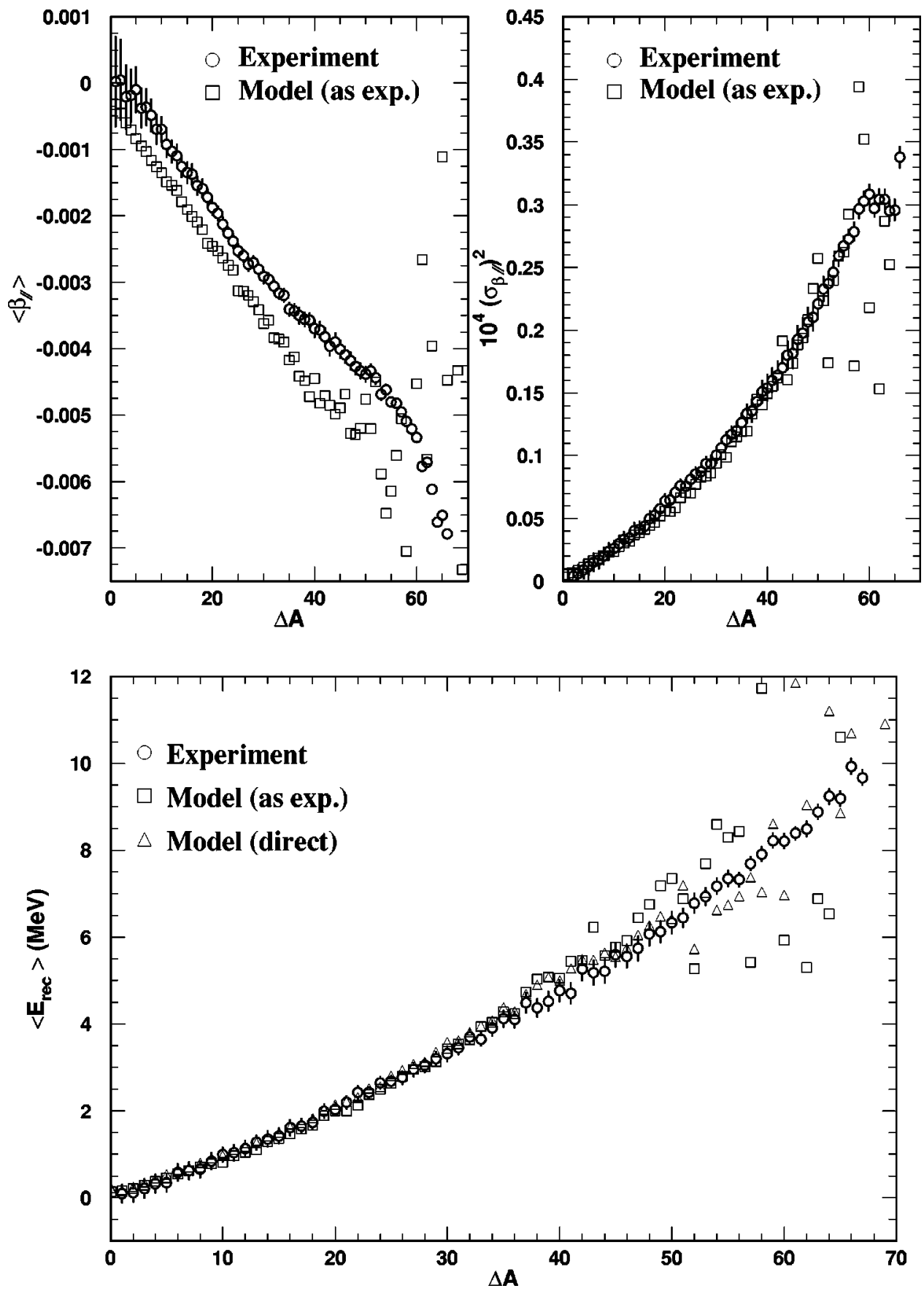

FIG. 21. The upper part of the figure gives the average value (left) and the variance (right) of the longitudinal velocity of the residue, relative to the one of the incident nucleus, as a function of the mass loss $\Delta A$. The figure refers to ${ }^{208} \mathrm{~Pb}$-induced reactions on a $\mathrm{H}$ target at $1.0 \mathrm{GeV}$ per nucleon incident energy. Data (circles), from Ref. [60], are compared to the predictions (squares) of the INCL4 + KHSv3p model, calculated using the same assumption as in the experiment. The lower part of the figure displays the average recoil energy of the residues in the rest frame of the incident ${ }^{208} \mathrm{~Pb}$ nucleus, for given mass loss. Data (circles) are compared with the predictions of the model (triangles). The squares give the predictions of the model, evaluated using the same reconstruction method as experimentally. See text for detail.

reactions than in proton-induced reactions at the same incident energy per nucleon, but less than twice as large. This property mainly arises from the fact that for large impact parameters, one of the nucleons of the deuteron does not interact. We can also compare deuteron-induced and protoninduced reactions at the same total incident energy. The particle multiplicities are surprisingly similar in the two cases. The near equality of the experimental neutron multiplicities was already pointed out in Ref. [68] for proton and ${ }^{3} \mathrm{He}$ induced reactions at the same total incident kinetic energy. We think that this results from the fact that in the limited range of energy under consideration the specific energy loss of a nucleon is roughly proportional to its energy [13]. Therefore roughly the same total energy loss appears for in- cident proton or deuteron at the same energy. The number of cascade particles are also roughly the same. The excitation energy does follow this simple rule a little bit more loosely.

\section{Residue production}

Our predictions are compared, in Figs. 23 and 24, with the experimental data of Ref. [69], for $\mathrm{Pb}+d$ reactions at $1 \mathrm{GeV}$ per nucleon. In this case, we overestimate the fission cross section, which might indicate that the fission model is perhaps not so well adapted to high excitation energy. Among all the cases investigated here, it is the one with the largest available energy, and thus the largest excitation energy. This is also the reason why the fragmentation peak is broader than 


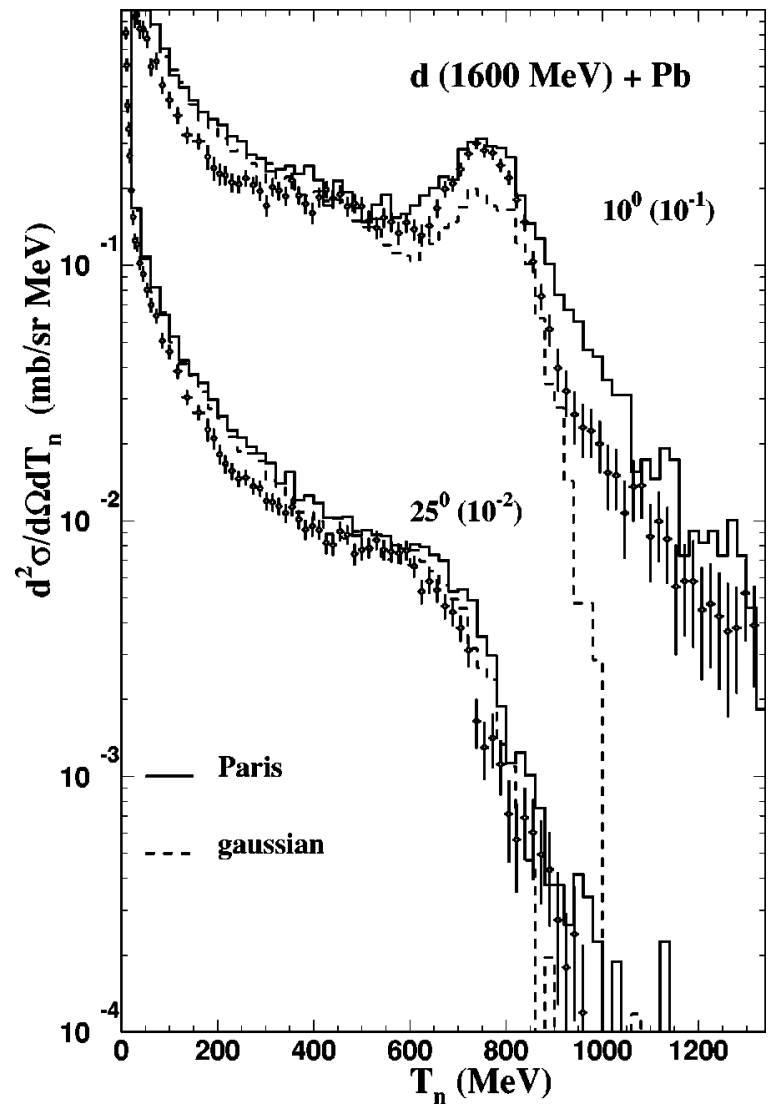

FIG. 22. Neutron double differential cross sections for deuteroninduced reactions on a $\mathrm{Pb}$ target at $800 \mathrm{MeV}$ per nucleon incident energy, plotted with a linear energy scale. The predictions of the INCL4 + KHSv3p model are given by the histograms. Two momentum distributions have been used for the deuteron: a Gaussian shape with variance given in Table I (dashed histogram) and deuteron wave function in momentum space, as calculated from the Paris potential (full histogram). Data (circles) are from Ref. [67]. Same convention as in Fig. 4.

in the other cases. Our results correctly reproduce this part of the spectrum, except, as before, in the low mass side. Figure 24 shows that the isotopic distributions are rather well reproduced. In particular, the isotopic distributions of the fission fragments are well reproduced except for their amplitude. Therefore the overestimate of the fission cross section is coming from the competition between evaporation and fission. A friction parameter varying with the excitation energy would probably accommodate this discrepancy.

TABLE IV. Mean particle multiplicities and mean excitation energy $\left\langle E^{*}\right\rangle$ (in $\mathrm{MeV}$ ) in proton-induced and deuteron-induced reactions on $\mathrm{Pb}$, as predicted by our model.

\begin{tabular}{lcccc}
\hline \hline & $\begin{array}{c}p+\mathrm{Pb} \\
800 \mathrm{MeV}\end{array}$ & $\begin{array}{c}p+\mathrm{Pb} \\
1600 \mathrm{MeV}\end{array}$ & $\begin{array}{c}d+\mathrm{Pb} \\
800 \mathrm{MeV}\end{array}$ & $\begin{array}{c}d+\mathrm{Pb} \\
1600 \mathrm{MeV}\end{array}$ \\
\hline$n E<2 \mathrm{MeV}$ & 3.33 & 3.44 & 3.28 & 3.50 \\
$n 2<E<20 \mathrm{MeV}$ & 6.83 & 8.88 & 6.45 & 9.40 \\
$n E>20 \mathrm{MeV}$ & 2.48 & 3.68 & 2.84 & 4.40 \\
$p E<20 \mathrm{MeV}$ & 0.48 & 0.84 & 0.43 & 0.93 \\
$p E>20 \mathrm{MeV}$ & 2.08 & 2.84 & 2.01 & 3.09 \\
$\left\langle E^{*}\right\rangle$ & 120 & 166 & 90 & 177 \\
\hline \hline
\end{tabular}

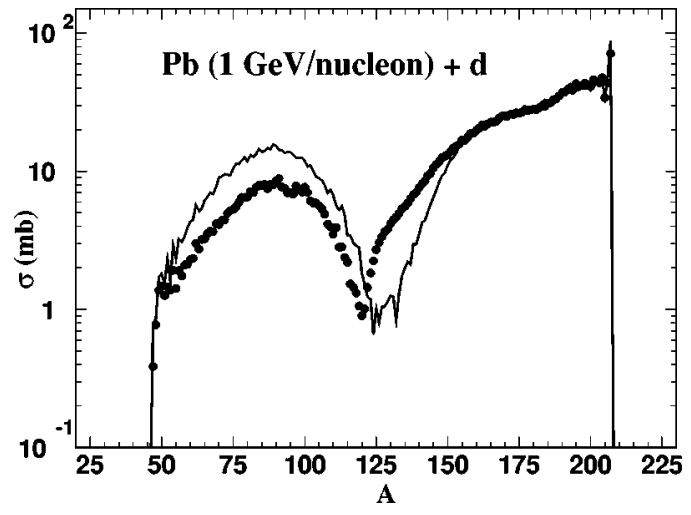

FIG. 23. Residue mass production cross sections for ${ }^{208} \mathrm{~Pb}$-induced reactions on a $D$ target at $1.0 \mathrm{GeV}$ per nucleon incident energy. The predictions of the INCL4 + KHSv3p model are given by the full line, including only the isotopes that have been measured experimentally. Data (dots) are from Ref. [69].

\section{VARIATION OF THE RESULTS WITH MODIFICATIONS OF THE MODEL}

\section{A. Modifications of the stopping time}

As stated in Sec. II, our new INC model has only two free parameters: the potential depth $V_{0}$ and the param-

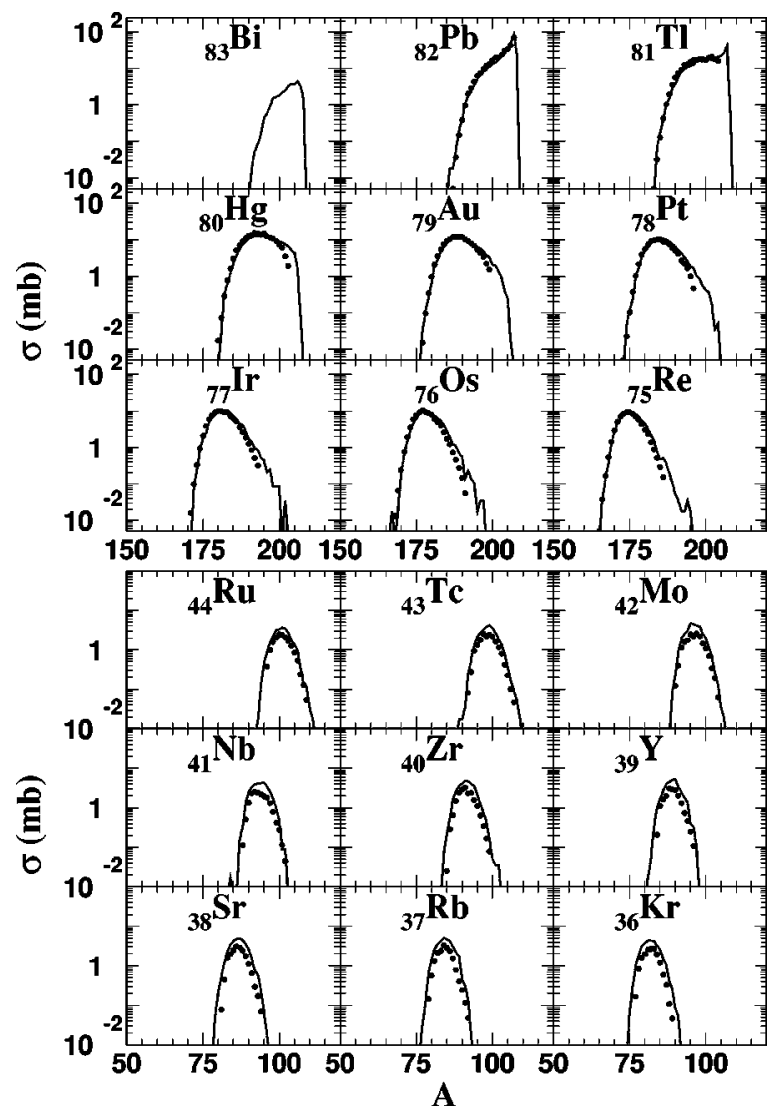

FIG. 24. Isotopic distributions of the nuclides produced in ${ }^{208} \mathrm{~Pb}$-induced reactions on a $D$ target at $1.0 \mathrm{GeV}$ per nucleon incident energy. Only the most representative nuclides are shown. Predictions of the INCL4 + KHSv3p model are given by full lines. Data (dots) are from Ref. [69]. 

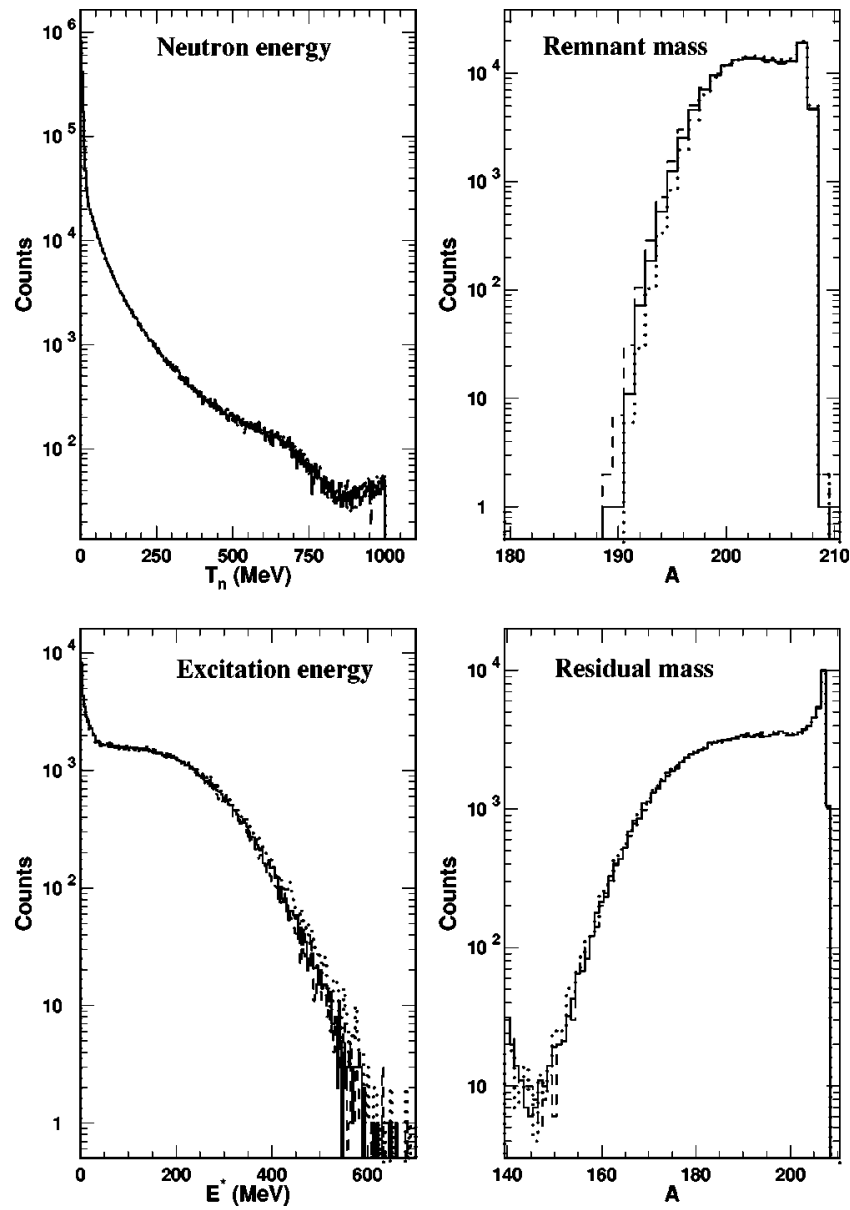

FIG. 25. Comparison of the results obtained with our INCL4 model, for the $p+\mathrm{Pb}$ system at $1 \mathrm{GeV}$, using three values for the stopping time, corresponding to $f_{\text {stop }}=0.9$ (dots), 1.0 (full line), and 1.1 (dashes) [see Eq. (29)]. The graphs represent the neutron energy spectrum, the remnant mass, the excitation energy distribution at the end of the cascade stage, and the final residue mass distribution, respectively.

eter $f_{\text {stop }}$, which controls the stopping time. As the value of the first one is tightly bound to the nucleon separation energy, we limit ourselves to investigate the influence of varying the stopping time. In Fig. 25, we compare physical quantities obtained with different values of the parameter $f_{\text {stop }}$, namely, $0.9,1.0$, and 1.1. The changes of the results are rather small. For instance, if one tries to quantify the change in the excitation energy distribution by the width at half maximum (of the plateau), the modification is less than 5\%. The remnant mass distribution appears to be slightly more sensitive, but the residue mass distribution shows a smaller sensitivity, similar to the one of the excitation energy distribution. The results of Fig. 25 indicate that there is a time interval just before the beginning of the evaporation process in which the excitation energy presents little variation. This legitimizes the notion of excitation energy left after the cascade process, as this quantity is defined within a few percent, even if it is hardly measurable with accuracy.

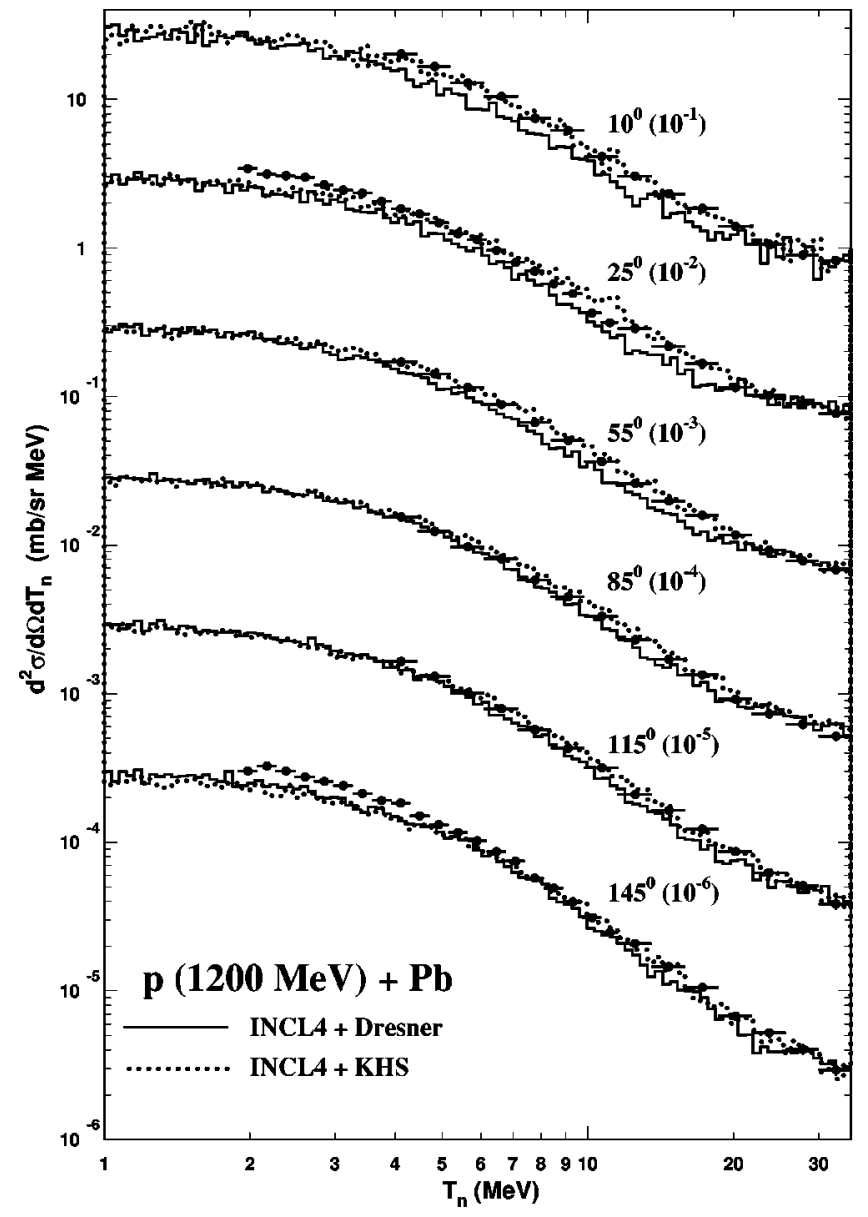

FIG. 26. Low energy neutron double differential cross sections for proton-induced reactions on a $\mathrm{Pb}$ target at $1.2 \mathrm{GeV}$ incident energy. Predictions of INCL4 associated with the Dresner (full histogram) or with the KHSv3p (dotted histogram) evaporation models are displayed. Data (circles) are from Ref. [19]. In order to ease the reading of the figure, the same convention as in Fig. 4 has been used.

\section{B. Sensitivity to the parameters of the Pauli blocking}

Although the parameters $r_{P B}$ and $p_{P B}$ [Eq. (8)] of the stochastic Pauli blocking are not free in the INCL4 code, we nevertheless checked the sensitivity of the results to the changes of $r_{P B}$. We found that decreasing its value down to $2 \mathrm{fm}$ does not change the results significantly, at least for residue production and particle multiplicities. Such a modification of the parameter reduces the size of the measuring volume from 2.3 to 0.5 natural phase-space units.

\section{Modifications of the evaporation model}

As we were not entitled to change the parameters of the Schmidt evaporation code, we investigate this point by employing another evaporation model, namely, the Dresner code [41] (with the default parameters). The results obtained with this evaporation code are given in Figs. 26-28 and Table III. The predictions for the evaporative part of the neutron double differential cross sections (Fig. 26) are basically 


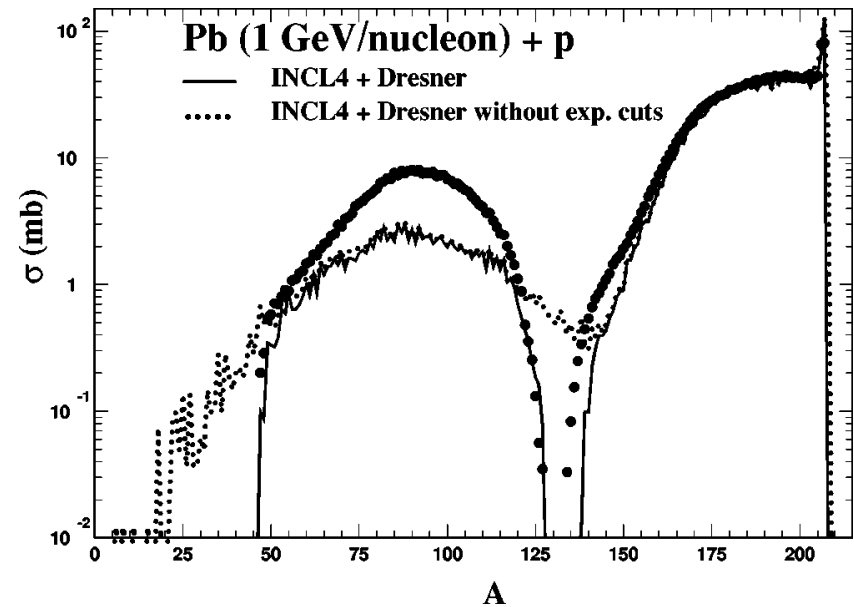

FIG. 27. Residue mass production cross sections for ${ }^{208} \mathrm{~Pb}$-induced reactions on a $\mathrm{H}$ target at $1.0 \mathrm{GeV}$ per nucleon incident energy. The predictions of the INCL4 + Dresner model are given by the lines, including all isotopes (dotted line) or only those that have been measured experimentally (full line). Data (dots) are from Ref. [60].

the same as with the KHSv3p model (the cascade part remains, of course, unchanged). They are slightly less good, showing a slightly steeper slope. These features are in keeping with the discussion of Sec. III A 2: the number of evaporated neutrons does not depend so much on the evaporation model, whereas the slope of the spectra, related to average temperature, is more sensitive.

Figure 27 shows that the predictions for the residue mass cross sections with the Dresner evaporation model are quite different from those of the KHSv3p model (compare with Fig. 15). We recall that these differences are solely due to the evaporation models, but of course this statement strictly applies to the very mass-excitation energy distribution generated by our cascade model. The Dresner model considerably underestimates the fission component. Also the width of the fission peak is broader with this model. On the other hand, the Dresner model reproduces much better the low mass (charge) side of the fragmentation peak.

It is instructive to look at the isotopic distributions generated by the two evaporation models (Figs. 28 and 16). For the Dresner model, the maxima lie systematically three or four units too far on the high mass side, as compared to experiment, for nuclides in the fragmentation peak. This may be attributed to a wrong competition between neutron and charged-particle evaporation which drives the residues too far to the neutron-rich side of the so-called residue corridor $[23,60]$, presumably as a result of too small Coulomb barriers. Indeed Table III shows that the evaporation $n / p$ ratio comes out with different values in the two models. If one also counts neutrons and protons contained in composites, the Dresner model evaporates, for example, in the $p(1.2 \mathrm{GeV})+\mathrm{Pb}$ case, 12.96 neutrons and 2.78 protons, whereas the KHSv3p model evaporates 12.75 neutrons and 1.92 protons. The $n / p$ ratio amounts to 4.66 and 6.64 , respectively. One can also notice from Fig. 28 that the isotopic distributions of the fission isotopes are characterized by a correct location of the maximum, but a wrong amplitude.
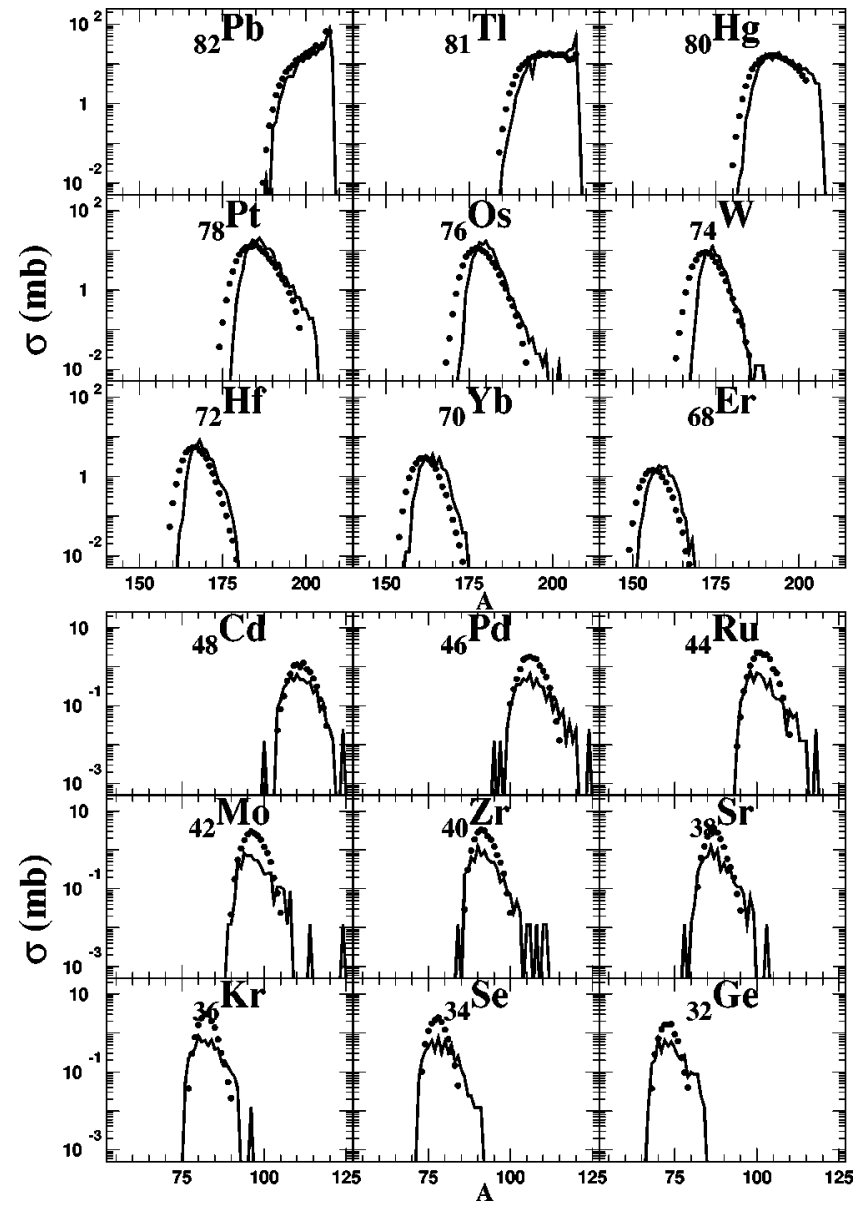

FIG. 28. Isotopic distributions of nuclides produced in ${ }^{208} \mathrm{~Pb}$-induced reactions on a $\mathrm{H}$ target at $1.0 \mathrm{GeV}$ per nucleon incident energy. Top and bottom panels refer to representative nuclides of the fragmentation and fission peaks, respectively. Predictions of the INCL4 + Dresner model are given by the full lines. Data (dots) are from Ref. [60].

The Dresner model thus seems to generate fission fragments with the correct charge-to-mass ratio.

We do not intend here to enter into the intricacies of the two evaporation models, but we want to comment a little bit on their differences. Both models use the Weisskopf-Ewing model for particle production and accommodates $n, p, \alpha$ evaporation. The Dresner model accommodates also $d$, $t$, and ${ }^{3} \mathrm{He}$ emission. As far as we know, the other main differences come from the behavior of the level parameter $a$ and from the barrier heights at high excitation energy: $a$ tends to $A / 8$ in the Dresner code and to $\sim A / 11$ in the KHSv3p one, proton Coulomb barriers are significantly reduced at high excitation energy in the Dresner code. This more or less explains why the Dresner code produces more protons (see Table III) and slightly more neutrons, with an effective smaller temperature (see Fig. 26), corresponding to a larger parameter $a$. Both models use the transition state method for the fission width. The neutron-fission competition is then mainly governed by the $a_{f}$ parameter, related to the level density at the saddle point. Angular momentum, which enhances fission, and friction, which reduces it, are included in the KHSv3p model and not in the Dresner one. In the latter, the fission model is 
the so-called RAL model revisited by Atchison [70]. We are unable to determine what ingredient is the most important for the better agreement reached by the KHSv3p model for the fission yield. Fission fragment mass distribution and the charge to mass ratio distribution are based on low energy phenomenology in the Dresner code. They are based on the properties of potential energy surfaces at the conditional saddle point in the KHSv3p case [43]. One can conclude that the latter approach is much better suited (concerning fission) to the excitation energy regime explored here. In particular, the width of the fission peak in residue mass spectra is coming from the enhancement of the stiffness of the potential curve along the mass asymmetry degree of freedom [71].

\section{DISCUSSION}

\section{A. Global interpretation of the results}

The overall agreement obtained by our INC + evaporation model for a large set of observables strongly supports the basic premise of the model, namely, the division of the interaction process in a first cascade stage, dominated by successive well separated collisions occurring basically like in free space, followed by a second stage in which the fairly equilibrated remnant evaporates particles or fissions. In addition, the fact that the excitation energy does not vary very rapidly with time at the end of the cascade process, when the remnant reaches equilibrium (or at least randomization), legitimizes the concept of a hot nucleus formed in spallation reactions in the $\mathrm{GeV}$ range.

\section{B. Single versus multiple scattering}

As we alluded to before, it is paradoxical that the agreement is definitely better for the multiple scattering component than for the single scattering one. These components are not directly measurable, but studies of the INC model indicate $[14,27]$ that single scattering component overwhelmingly dominates the quasielastic peak (and part of the quasiinelastic peak) in neutron or proton energy spectra and the $\left(A_{T}, Z_{T}-1\right)$ and $\left(A_{T}, Z_{T}\right)$ yields in residue spectra. The multiple scattering component corresponds roughly to the interval $\left(20 \mathrm{MeV}, T_{l a b} / 2\right)$ for particle spectra and to the part of the fragmentation peak in mass spectra including residues with charge (mass) number a few units off the target charge (mass) number. As we said in Sec. III, the most plausible explanation of the above-mentioned paradox is that the quantum, collective and possible medium effects neglected in our quasiclassical approach are likely to fluctuate from any one elementary scattering to the other and that these effects are more or less washed out in the many-scattering contribution.

Detailed studies of the quasielastic scattering, for which the single-scattering contribution is dominant, revealed complex features [51]. The shape of the peak is governed by the one particle-one hole phase space, as expected. The position of the peak is shifted toward large energy losses compared to the quasifree kinematics, by $20-30 \mathrm{MeV}$, in $(p, n)$ reactions, more or less independently of the target mass and of the momentum transfer [this is also true for $\left(e, e^{\prime}\right)$ and $\left({ }^{3} \mathrm{He}, t\right)$ reactions]. On the other hand, the peak is not shifted in $\left(p, p^{\prime}\right)$ reactions. The amplitude of the peak is not reproduced by current single-scattering models. Most of them, based on the Glauber approach, underestimate it badly, even when double scattering is included. The latter process turns out to be small anyway, as confirmed by INC calculations [27]. Quantum-mechanical approaches to the quasielastic scattering express the cross sections in terms of the transition probability for passing from the original ground state to any final state consistent with a given momentum transfer in a single interaction, and of the probability that there is neither a prior nor a post interaction. This latter probability is usually translated into the so-called effective number of interacting target nucleons $N_{e f f}$, whose evaluation often rests on a Glauber picture. The fact that these approaches usually underestimate the intensity of the quasielastic peak, whereas our model gives reasonable values, induces us to believe that the Glauber approach is rather naive for the evaluation of $N_{\text {eff }}$. The INC model is expectedly much more appropriate for taking proper account of the actual geometry of the collision process. The shift of the quasielastic peak is believed to arise from the combination of collective effects, which distort the $1 p-1 h$ strength compared to the Fermi-gas one, and of the spin-dependence of the nucleon-nucleon interactions $[72,73]$. The spin-longitudinal and spin-transverse responses seem to behave differently [48] and sum up differently in $(p, n)$ and $\left(p, p^{\prime}\right)$ channels. Our model contains neither collective effects nor a spin dependence of the nucleon-nucleon interaction. It is then natural that our predicted location of the quasielastic peak coincides with the quasifree estimate and agrees with experimental data in the case of $\left(p, p^{\prime}\right)$ reactions (see Fig. 12).

\section{Shape of the fragmentation peak}

It has been repeatedly attempted to relate qualitatively the shape of the fragmentation peak to the distribution of the excitation energy, and the latter to the basic input of the INC model, namely, the salient features of nucleon-nucleon collisions [74-77]. We follow here the presentation of Ref. [76] and compare it to our results. In this reference, the authors assume that the evaporation of a nucleon consumes a constant value $\epsilon$ of excitation energy and neglect the mass loss in the cascade stage. They are able to express the mass yield as

$$
\sigma(A)=\left.\epsilon \frac{d \sigma}{d E^{*}}\right|_{E^{*}=\left(A_{T}-A\right) \epsilon} .
$$

They also argue that the excitation energy distribution has an exponential form

$$
\frac{d n}{d E^{*}} \propto \exp \left[-\frac{E^{*}}{E_{0}^{*}}\right],
$$

although their estimate of $E_{0}^{*}$ is not very realistic (see Ref. [13] for a comparison). Using this equation, one gets 

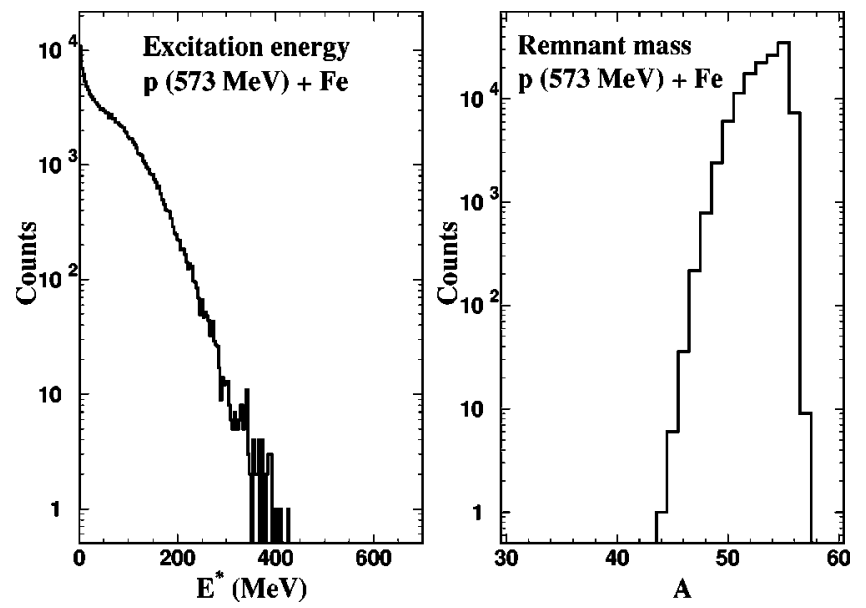

FIG. 29. Calculated excitation energy (left) and remnant mass (right) distributions after the cascade stage for proton-induced reactions on a ${ }^{56} \mathrm{Fe}$ target at $573 \mathrm{MeV}$ incident energy.

$$
\sigma(A)=\sigma_{R}^{t o t} \frac{\epsilon}{E_{0}^{*}} \exp \left(-\frac{\epsilon}{E_{0}^{*}}\left(A_{T}-A\right)\right)
$$

This is an amazingly simple form. Unfortunately, it does not agree with the data displayed in Figs. 15 and 17. The basic reason comes from the supposedly exponential form for the excitation energy distribution, which is not very realistic as shown by Fig. 25: a shoulder develops at high excitation energy. The latter comes from small and intermediate impact parameters and is due, in our opinion, to secondary collisions, whose importance is not reducible to such simple considerations as in Ref. [76]. For light targets and small incident energy, these effects are of lesser importance, and a decreasing exponential is not a bad approximation for the $E^{*}$ distribution, as corroborated by Fig. 29. This is consistent with the exponential shape of the mass spectrum for $\mathrm{Fe}+p$ collisions at $573 \mathrm{MeV}$ per nucleon (Fig. 19). For protoninduced collisions on $\mathrm{Pb}$ at $1 \mathrm{GeV}$ (Fig. 15), the mass yield (in the fragmentation peak) can be qualitatively understood, just by considering the excitation-energy distribution of Fig. 25 and the argument leading to Eq. (32). The shapes of the fragmentation peak and of the theoretical excitation distribution are indeed strikingly similar.

\section{Further improvements of the model}

Although we achieve a good agreement with most of the experimental data, the model still suffers from some weaknesses. We here quote a few possible further improvements inside the basic framework of the quasiclassical approach of nuclear multiple scattering:

(i) As we do for the spatial density, one should use a realistic momentum density, which, in actual nuclei, may differ sizably from the Fermi gas density [78].

(ii) It has been known for a long time that the nuclear mean field is nonlocal or, more or less equivalently, momentum-dependent $[79,80]$. This may be of importance as the incident particle experiences practically no field when its energy exceeds $\sim 200 \mathrm{MeV}$. (iii) Pion dynamics should be improved. This is an important point, as we overestimate the pion yield (see Fig. 13) and therefore also the energy flow carried by these particles. The most obvious remedy is provided by the discussion of the next item.

(iv) In-medium effects should be introduced. The most documented aspect of these effects stems for the nucleonnucleon elastic scattering, since the correction due to short range correlations has been studied by several authors, mainly in the context of Brueckner theory $[55,81,82]$. This modification brings, however, only minor effects [14], except perhaps at low incident energy [83]. On the contrary, the cross sections involving $\Delta$ particles may be changed drastically by these short-range correlations [55]. Effects of similar size are expected for other quantities like the in-medium delta and pion masses.

(v) Another missing feature in our approach is the production of composites during the cascade stage, although these particles are much more abundantly produced by evaporation. Introduction of composite degrees of freedom and their interaction with nucleon degrees of freedom within the nucleus is a delicate matter. A simple approach consists in applying a percolation procedure at the end of the cascade stage $[84,85]$ or at the nuclear surface for outgoing particles [86].

\section{COMPARISON WITH OTHER WORKS}

Let us first consider other INC+evaporation models. Probably the most utilized model of this kind is the Bertini model [16], perhaps because it is included as the standard choice in the LAHET code system [87], which aims at describing high-energy particle transport in matter. The Bertini model differs from ours in many respects. First, in this model, the target is seen as a continuum providing the traveling particles with a mean free path. At the end of a path, taken at random, the traveling particle is forced to collide with a nucleon, promoted as a new traveling particle from the continuum. Second, the cascade process is stopped when the energies of all particles inside the target are lower than a given value, considered as a parameter. Third, the density profile is assumed to be given by three uniform spheres. Fourth, parametrization of inelastic cross sections is rather crude. Comparison between the predictions of the Bertini code and of the INCL2 version of our code has been done on several occasions. In particular, it has been shown that our code is systematically better for neutron double differential cross sections, as measured at SATURNE [19,21]. In particular the Bertini model presents some pathological behavior in the quasi-inelastic region, due to a too simplified model for delta production. The evaporation part of the spectra is slightly overestimated by the Bertini code. This can be attributed to the INC Bertini model, as the same Dresner evaporation model is used in the two cases. For the rest of the phase space, our INCL2 model produces better results, except at intermediate angles (around $90^{\circ}$ ) and large neutron energy.

Globally, the results with INCL4 (Figs. 5-7) are slightly less good than with the INCL2 (except in the quasielastic 
region, as mentioned in Sec. III), but still better than those of the Bertini model. Concerning the integrated neutron spectra (neutron multiplicity), a partial comparison is provided by Table II. The predictions of our model are given in the third column. The fourth and fifth columns give the predictions of INCL2 and Bertini models, with the same Dresner evaporation code, corrected this time for the thickness of the target $(2 \mathrm{~cm})$, using the TIERCE [88] and the LAHET transport codes, respectively. As we already mentioned, this correction diminishes the neutron multiplicity for $E>20 \mathrm{MeV}$ by $\sim 0.2-0.3$ units (increasing with the incident energy) and leaves the $2-20-\mathrm{MeV}$ multiplicities practically unchanged. One can see from Table II than our model gives slightly better results than the Bertini model for the above $20 \mathrm{MeV}$ multiplicities and much better results in the $2-20-\mathrm{MeV}$ interval.

We want also to shortly compare with the ISABEL INC model $[17,18]$, another popular numerical code. The latter differs from the Bertini code by refinements of the target density, of the elementary cross section parametrizations, of the delta production scenario and by several other options concerning especially the rearrangement of the continuum and the treatment of the Pauli principle. This model gives results similar to ours for the total reaction cross section [87], for neutron double differential cross sections and also for residue mass spectra [89]. However, it seems that the ISABEL model produces less satisfactory results for lighter targets [21]. Let us also mention that other recent works $[90,91]$ seem to yield good results comparable to ours, but to the best of our knowledge, no comprehensive calculation with fixed parameters (like in this paper) has been published for these models.

It has often been advocated that the division of the collision process into two stages is not a satisfactory feature, because one expects a smooth evolution from a cascade into an evaporation dynamics, and that the introduction of a socalled pre-equilibrium stage between cascade and evaporation would alleviate this weakness. Initially, pre-equilibrium models have been used to describe reactions in the $\sim 100-\mathrm{MeV}$ incident energy regime. There are several versions of them. See Refs. [92,93] for a review. They have been introduced in a three step (cascade + pre-equilibrium + evaporation) model for spallation reactions by Mashnik and his group [94,95]. Roughly speaking, the cascade stage is stopped when the particles have still a few tens of $\mathrm{MeV}$; then the particles are placed on some states of a singleparticle spectrum, representative of the shell model of the nucleus, according to their energy. The occupation numbers of the single-particle states are assumed to change, simulating the effects of collisions, with probabilities given by the usual mean-free-path considerations. In some sense, this stage can be viewed as a cascade model in a discrete energy space. Finally, when the occupation number distribution is close to a thermal one, a standard evaporation step is introduced. There are indications that the introduction of such a step is perhaps not necessary or appropriate. First of all, the data for which such an intermediate step is expected to show effects are well described by a two-step model like ours. The most prominent example is the neutron spectra in the 15-40-
$\mathrm{MeV}$ range, which corresponds to the transition between cascade and evaporation production. Figures 5-7, for instance, do not reveal any particular deficiency of our approach in this range. Second, one should question the argument that a discrete energy space should be preferred to the continuum phase space to account for quantization when the energy of the nucleons becomes sufficiently small, i.e., comparable with the energy difference between major shells. In fact, interactions here may help to disregard a discrete singleparticle spectrum. Indeed, interacting nucleons are significantly off shell $[79,96]$ : this means that their energy is not precisely defined for a given momentum or vice versa. This feature is expected to smear out the effects of the quantization of energy and to justify to some extent the quasiclassical approach with a continuous phase space. Third, as we discussed in Sec. II, the INC model is doing itself the transition up to thermalization of the remnant (see Fig. 2). Of course, continuing with the INC model to describe evaporation would not be correct. This model is an independent-particle model, whereas evaporation models implicitly introduce strong correlations: this is reflected by the level-density parameter $a$ which is usually taken to be quite different from the pure Fermi gas value A/16. That is why one should switch off the cascade at some time, besides the fact that describing the evaporation by an INC model would require long computational times. Fourth, introduction of a preequilibrium stage may give accurate results for the description of the neutron spectra depending upon the associated cascade model (see Table II, last column, and Refs. [14,89]), but it seems that these models are working with different degrees of success for different target mass regions [21]. Let us stress, however, that such three-step models are well suited to describe the direct emission of composite particles with energy larger than $\sim 20 \mathrm{MeV}$. For that particular point, there is presently no other valuable competing model.

\section{CONCLUSION}

Compared to the previous ones, the latest version of the Liège INC model presented here includes mandatory physical aspects, the most important being the introduction of a diffuse nuclear surface and a consistent handling of the Pauli blocking effect. The numerical code INCL4, an implementation of this model, can handle nucleon-induced as well as cluster-induced reactions (for $d, t,{ }^{3} \mathrm{He}$, and ${ }^{4} \mathrm{He}$ clusters) on the same footing (and also pion-induced reactions, not covered in this publication). The remnant angular momentum is now included in the output of the model.

This new version has been tested successfully, in the 200$\mathrm{MeV}-2-\mathrm{GeV}$ range, against a large data base, including total reaction cross sections, neutron, proton, and pion double differential cross sections, alpha spectra (in the evaporation range), residue production cross sections, and residue recoil energy distributions. For comparison with data requiring an evaporation model, we here chose the KHSv3p version of the Schmidt model. Although a large part of the data provides a mixed test of the two models, whose respective contributions it is sometimes difficult to disentangle, we have nonetheless isolated in many occasions the INC contribution. 
We stress that the good agreement reached in this work has been obtained with a single set of the two free parameters of the INCL4 model (and without varying the parameters of the KHSv3p model), and this for a large set of data covering many different observables.

Our work was motivated by (i) an attempt to improve the understanding of the physics of spallation reactions and the apparent absence of quantum effects, except for Pauli blocking and the stochastic nature of the elementary collisions; (ii) the advent of good quality data for neutron spectra and especially for residue production; (iii) the necessity of having suitable simulation tools for technological applications (ADS, neutron spallation sources, e.g.). The good agreement obtained in this work is definitely helpful with respect to the first point. Concerning the third point, we are unable for the moment to state whether or not our model has a sufficient accuracy. The conception of an ADS or even of a spallation source requires so many studies, of which the output of the spallation reactions is only one ingredient among many others. There is presently no available extensive sensitivity studies concerning the influence of the spallation data on the performances of an ADS design. Some efforts [97-99] are currently done concerning the influence of the spallation data on the energy spectrum and angular distribution of neutrons emitted from a thick target bombarded by high energy protons, for which high-accuracy data have recently been obtained at SATURNE [100] and at FZ Jülich [101].

The philosophy supporting this work was to describe as well as possible the known physics of spallation reactions without resorting to too many free parameters. This program is not complete and improvements within this framework are still possible. We have, however, the impression that, if the accuracy of the results should be increased to satisfy the future requirements of technological applications, this would not be possible without the introduction of extra free parameters in order to mock up physical effects that are hard to handle in a simple and consistent model. The collective effects suspected in the quasi-elastic region of neutron spectra provides a nice example of what we have in mind.

We think that the good results of our model are substantially due to the "self-consistent" choice of the stopping time, which allows us to include all the collision dynamics until the remnant is largely equilibrated. We produced various arguments supporting this view. In addition, the fact that the excitation energy of the remnant is slowly varying at this time somehow legitimizes the concept of a hot nucleus and the relevance of its excitation energy. The precise measurement of this quantity is still, in our opinion, not possible. Even if calorimetry measurements are envisaged, this would require the simultaneous measurements of all low energy particles. Experiments with such a goal have recently been proposed $[102,103]$.

\section{ACKNOWLEDGMENTS}

This work has been done as a participation in the HINDAS collaboration (European Union Contract No. FIKWCT-2000-00031). We acknowledge the EU financial support. We are grateful to our HINDAS colleagues for in- teresting discussions and particularly to Dr. Karl-Heinz Schmidt for providing us with his evaporation code. One of the authors (J.C.) is very thankful for the kind hospitality extended to him during his stay at the SPhN-DAPNIA of the CEA at Saclay, where most of this work has been performed.

\section{APPENDIX: DEMONSTRATION OF EQS. (6) AND (7)}

We demonstrate that the joint distribution [Eq. (5)] generates the density profile [Eq. (6)] and the momentum distribution [Eq. (7)]. We first verify that $R\left(p_{F}\right)=R_{\max }$ follows from the definition of $R(p)$ by Eq. (4). Indeed performing the integration in Eq. (4) by parts yields, for $p=p_{F}$ :

$$
1=-\frac{4 \pi}{3 A_{T}}\left(\rho\left(R_{\max }\right) R_{\max }^{3}-3 \int_{0}^{R_{\max }} \rho(r) r^{2} d r\right) .
$$

If $R_{\max }$ is large enough, the first term in the parenthesis vanishes and the relation just expresses the normalization of the density $\rho(r)$ to $A_{T}$.

Equation (7) is obtained after a straightforward integration of Eq. (5) over the variable $\vec{r}$. For the demonstration of Eq. (6), we consider the integration over $\vec{p}$ of the two sides of Eq. (5),

$$
F(r)=\int f(\vec{r}, \vec{p}) d^{3} \vec{p}=\frac{4 \pi A_{T}}{\left(\frac{4 \pi}{3}\right)^{2} p_{F}^{3}} \int_{0}^{p_{F}} \frac{\theta[R(p)-r] p^{2} d p}{R^{3}(p)}
$$

and the derivative of $F$

$$
\begin{aligned}
\frac{d F}{d r} & =-\frac{4 \pi A_{T}}{\left(\frac{4 \pi}{3}\right)^{2} p_{F}^{3}} \int_{0}^{p_{F}} \frac{\delta[r-R(p)] p^{2} d p}{R^{3}(p)} \\
& =-\frac{4 \pi A_{T}}{\left(\frac{4 \pi}{3}\right)^{2} p_{F}^{3}}\left(\frac{p^{2}}{R^{3}(p)} \frac{1}{\left|R^{\prime}(p)\right|}\right)_{p=R^{-1}(r)},
\end{aligned}
$$

where $R^{-1}$ is the inverse function of $R(p)$. Taking the derivative of Eq. (4), which defines $R(p)$, we can write

$$
3 \frac{p^{2}}{p_{F}^{3}}=-\frac{4 \pi}{3 A_{T}}\left(\frac{d \rho(r)}{d r} r^{3}\right)_{r=R(p)} R^{\prime}(p) .
$$

This equation can be rearranged and, for $p=R^{-1}(r)$, yields 


$$
\left(\frac{p^{2}}{R^{3}(p) R^{\prime}(p)}\right)_{p=R^{-1}(r)}=-\frac{4 \pi p_{F}^{3}}{9} \frac{d \rho(r)}{d r},
$$

using $R\left[R^{-1}(r)\right]=r$. Combining this equation with Eq. (A3) and taking account of the positiveness of $R^{\prime}(p)$, we finally obtain

$$
\frac{d F}{d r}=\frac{d \rho}{d r}
$$

and, since $F\left(R_{\max }\right)=0$ according to Eq. (A2),

$$
F(r)=\rho(r) .
$$

[1] C. D. Bowman et al., Nucl. Instrum. Methods Phys. Res. A 320, 336 (1992).

[2] C. Rubbia et al., preprint CERN/AT/95-44 (ET), 1995.

[3] D. Filges, in Proceedings of the Fifth Workshop on Simulating Accelerator Radiation Environments (SARE-5), edited by D. Filges, F. Goldenbaum, and Y. Yariv (Jülich Publications, Jülich, 2001), p. 3.

[4] I. Tanihata, in Treatise on Heavy-Ion Science, edited by D. A. Bromley (Plenum Press, New York, 1989), Vol. 8.

[5] T. K. Gaiser, Cosmic Rays and Particle Physics (Cambridge University Press, Cambridge, England, 1992).

[6] M. Longair, High Energy Astrophysics, (Cambridge University Press, Cambridge, England, 1997), Vols. 1 and 2.

[7] R. C. Reedy and J. R. Arnold, J. Geophys. Res. 77, 537 (1972).

[8] A. Koning et al., International Conference on Nuclear Data for Science and Technology, Tsukuba, Japan, Oct. 7-12, 2001.

[9] J. Cugnon, Nucl. Phys. A387, 191c (1982).

[10] A. S. Iljinov, M. V. Kazarnovsky, and E. Ya. Paryev, Intermediate Energy Nuclear Physics (CRC Press, Boca Raton, 1994).

[11] J. Cugnon, T. Mizutani, and J. Vandermeulen, Nucl. Phys. A352, 505 (1981).

[12] J. Cugnon, P. Deneye, and J. Vandermeulen, Nucl. Phys. A500, 701 (1989).

[13] J. Cugnon, Nucl. Phys. A462, 751 (1987).

[14] J. Cugnon, C. Volant, and S. Vuillier, Nucl. Phys. A620, 475 (1997).

[15] J. Cugnon and P. Henrotte, in Proceedings of the Fifth Workshop on Simulating Accelerator Radiation Environments (SARE-5), edited by D. Filges, F. Goldenbaum, and Y. Yariv (Jülich Publications, Jülich, 2001), p. 65.

[16] H. W. Bertini, Phys. Rev. 131, 1801 (1963).

[17] Y. Yariv and Z. Fraenkel, Phys. Rev. C 20, 2227 (1979).

[18] Y. Yariv and Z. Fraenkel, Phys. Rev. C 24, 488 (1981).

[19] X. Ledoux et al., Phys. Rev. Lett. 82, 4412 (1999).

[20] M. Enke et al., Nucl. Phys. A657, 317 (1999).

[21] S. Leray et al., Phys. Rev. C 65, 044621 (2002).

[22] J. Benlliure et al., Nucl. Phys. A700, 469 (2002).

[23] W. Wlazlo et al., Phys. Rev. Lett. 84, 5736 (2000).

[24] C. Toccoli, Ph.D. thesis, University of Orsay, 2000.

[25] J. Aichelin, Phys. Rep. 202, 233 (1991).

[26] M. Durand, M. Brack, and P. Schuck, Z. Phys. A 286, 381 (1978).

[27] S. Vuillier, Ph.D. thesis, Paris-Sud University, France, 1998.

[28] J. Cugnon, S. Leray, E. Martinez, Y. Patin, and S. Vuillier,
Phys. Rev. C 56, 2431 (1997).

[29] J. Cugnon and M.-C. Lemaire, Nucl. Phys. A489, 505 (1988).

[30] C. Ciofi degli Atti, Nucl. Phys. A543, 183c (1992).

[31] R. B. Wiringa, Phys. Rev. C 43, 1585 (1991).

[32] J. Carlson, Phys. Rev. C 38, 1879 (1988).

[33] R. Schiavilla, V. R. Pandharipande, and R.B. Wiringa, Nucl. Phys. A449, 219 (1986).

[34] M. Lacombe, B. Loiseau, J.-M. Richard, R. Vinh Mau, J. Coté, P. Pirès, and R. de Tourreil, Phys. Rev. C 21, 861 (1980).

[35] The small peak in the $d E^{*} / d t$ curve at $t \sim 10 \mathrm{fm} / c$ is due to the partial transformation of the available energy into pions [see Eq. (15) and the remark about $W_{\pi}$ ].

[36] L. Pienkowski et al., Acta Phys. Pol. B 25, 732 (1994).

[37] R. E. Prael and M. B. Chadwick, Los Alamos National Laboratory preprint LA-UR-97-1744, 1997.

[38] B. C. Barashenkov, Cross-Sections of Interactions of Particles and Nuclei with Nuclei (JINR Publications, Dubna, 1993).

[39] J.-J. Gaimard and K.-H. Schmidt, Nucl. Phys. A531, 709 (1991).

[40] A. R. Junghans, M. de Jong, H. G. Clerc, A. V. Ignatyuk, G. A. Kudyaev, and K.-H. Schmidt, Nucl. Phys. A629, 635 (1998).

[41] L. Dresner, Oak Ridge report ORNL-TM-196, 1962.

[42] V. F. Weisskopf and D. H. Ewing, Phys. Rev. 57, 472 (1940).

[43] J. Benlliure, A. Grewe, M. de Jong, K.-H. Schmidt, and S. Zhdanov, Nucl. Phys. A628, 458 (1998).

[44] B. Jurado, A. Heinz, A. Junghans, K. -H. Schmidt, J. Benlliure, T. Enqvist, and F. Rejmund, preprint GSI 2001-14 .

[45] W. B. Amian et al., Nucl. Sci. Eng. 102, 310 (1989).

[46] S. Stamer et al., Phys. Rev. C 47, 1647 (1993).

[47] W. B. Amian et al., Nucl. Sci. Eng. 115, 1 (1993).

[48] W. M. Alberico, M. Ericson, and A. Molinari, Nucl. Phys. A379, 449 (1982).

[49] M. Ichimura, K. Kawahigashi, T. S. Jørgensen, and C. Gaarde, Phys. Rev. C 39, 1446 (1989).

[50] H. Esbensen and G. F. Bertsch, Phys. Rev. C 32, 553 (1985).

[51] D. L. Prout et al., Phys. Rev. C 52, 228 (1995).

[52] R. E. Chrien et al., Phys. Rev. C 21, 1014 (1980).

[53] J. A. McGill, G. W. Hoffmann, M. L. Barlett, R. W. Fergerson, E. C. Milner, R. E. Chrien, R. J. Sutter, T. Kozlowski, and R. L. Stearns, Phys. Rev. C 29, 204 (1984).

[54] D. R. F. Cochran et al., Phys. Rev. D 6, 3085 (1972).

[55] B. ter Haar and R. Malfliet, Phys. Rev. C 36, 1611 (1987).

[56] C. -M. Herbach et al., in Proceedings of the Fifth Workshop on Simulating Accelerator Radiation Environments 
(SARE-5), edited by D. Filges, F. Goldenbaum, and Y. Yariv (Jülich Publications, Jülich, 2001), p. 7.

[57] R. Michel et al., Nucl. Instrum. Methods Phys. Res. B 113, 429 (1996); 113, 439 (1996).

[58] M. Gloris, R. Michel, F. Sudbrock, U. Herpers, P. Malmborg, and B. Holmqvist, Nucl. Instrum. Methods Phys. Res. A 463, 593 (2001).

[59] H. U. Wenger et al., Ann. Nucl. Energy 26, 141 (1999).

[60] T. Enqvist et al., Nucl. Phys. A686, 481 (2001).

[61] B. Mustapha, Ph.D. thesis, University of Orsay, 1999.

[62] M. de Jong, A. V. Ignatyuk, and K.-H. Schmidt, Nucl. Phys. A613, 435 (1997).

[63] F. Rejmund et al., Nucl. Phys. A683, 540 (2001).

[64] J. Benlliure et al., Nucl. Phys. A683, 513 (2001).

[65] W. R. Webber, J. C. Kish, J. M. Rockstroh, Y. Cassagnou, R. Legrain, A. Soutoul, O. Testard, and C. Tull, Astrophys. J. 508, 940 (1998); 508, 949 (1998).

[66] R. Michel et al., Nucl. Instrum. Methods Phys. Res. B 103, 429 (1995); 103, 183 (1995).

[67] P. Pras et al. (unpublished).

[68] X. Ledoux et al., Phys. Rev. C 57, 2375 (1998).

[69] T. Enqvist et al., Nucl. Phys. A703, 435 (2002).

[70] F. Atchison, Intermediate Energy Nuclear Data: Models and Codes, Proceedings of a Specialists' Meeting (OECD/NEA Publications, Issy-les-Moulineaux, France, 1994), p. 199.

[71] P. Armbruster et al., Fragmentation of lead by $1 \mathrm{GeV}$ protons. Adapted model calculation for the production of residual nuclides, HINDAS report, 2001.

[72] V. R. Pandharipande, J. Carlson, S. C. Pieper, R. B. Wiringa, and R. Schiavilla, Phys. Rev. C 49, 789 (1994).

[73] J. Wambach, Phys. Rev. C 46, 807 (1992).

[74] S. B. Kaufman and E. P. Steinberg, Phys. Rev. C 22, 167 (1980).

[75] X. Campi and J. Hüfner, Phys. Rev. C 24, 2199 (1981).

[76] A. Y. Abul-Magd, W. A. Friedman, and J. Hüfner, Phys. Rev. C 34, 113 (1986).

[77] K. Sümmerer, W. Brüchle, D. J. Morrissey, M. Schädel, B. Szweryn and Yang Weifan, Phys. Rev. C 42, 2546 (1990).

[78] S. Frullani and J. Mougey, Adv. Nucl. Phys. 14, 1 (1984).

[79] J.-P. Jeukenne, A. Lejeune, and C. Mahaux, Phys. Rep. 25C, 83 (1976).

[80] A. E. S. Green, T. Sawada, and D. S. Saxon, The Nuclear
Independent Particle Model (Academic Press, New York, 1968).

[81] J. Cugnon, A. Lejeune, and P. Grangé, Phys. Rev. C 35, 861 (1981).

[82] G. Q. Li and R. Machleidt, Phys. Rev. C 49, 566 (1994).

[83] H. Takada, J. Nucl. Sci. Technol. 33, 275 (1996).

[84] J. Cugnon and C. Volant, Z. Phys. A 334, 435 (1989).

[85] D. Doré et al., Phys. Rev. C 63, 034612 (2001).

[86] A. Letourneau, Ph.D. thesis, University of Caen, 2000.

[87] R. E. Prael and H. Liechtenstein, Los Alamos National Laboratory report LA-UR-89-3014, 1989.

[88] O. Bersillon, in Second International Conference on Accelerator Driven Transmutation Technologies and Applications, edited by H. Condé (Gotlab, Stockholm, 1997), p. 520.

[89] S. Leray, A. Boudard, J. Cugnon, R. Legrain, and C. Volant, in Proceedings of the Monte Carlo 2000 Conference, edited by A. Kling, F. Barão, M. Nakagawa, L. Tàvora, and P. Vaz (Springer-Verlag, Berlin, 2001), p. 1111.

[90] K. Niita, International Conference on Nuclear Data for Science and Technology, Tsukuba, Japan, 2001.

[91] S. Furihata and T. Nakamura, International Conference on Nuclear Data for Science and Technology, Tsukuba, Japan, 2001.

[92] M. Blann, Annu. Rev. Nucl. Sci. 25, 123 (1975).

[93] E. Gadioli and P. E. Hodgson, Pre-Equilibrium Nuclear Reactions (Clarendon, Oxford, 1992).

[94] K. K. Gudima, S. G. Mashnik, and V. D. Toneev, Nucl. Phys. A401, 329 (1983)

[95] S. G. Mashnik and A. J. Sierk, invited talk to the International Conference on Nuclear Data for Science and Technology, Tsukuba, Japan, 2001; Los Alamos preprint LANL Report LA-UR-01-5390.

[96] O. Benhar, A. Fabrocini, and S. Fantoni, Nucl. Phys. A505, 267 (1989)

[97] L. Donadille et al., International Conference on Nuclear Data for Science and Technology, Tsukuba, Japan, 2001.

[98] D. Filges et al., Eur. Phys. J. A 11, 467 (2001).

[99] C. Varignon, Ph.D. thesis, University of Caen, 1999.

[100] S. Ménard, Ph.D. thesis, University of Orsay, 1997.

[101] A. Letourneau et al., Nucl. Instrum. Methods Phys. Res. B 170, 299 (2000).

[102] J. -E. Ducret et al., proposal S248, GSI, 2000.

[103] D. Filges et al., PISA experiment proposal, FZ Jülich, 2001. 